Philipp Brugger/Andreas Hasenclever/Lukas Kasten

\title{
Vertrauen lohnt sich
}

Über Gegenstand und Potential eines vernachlässigten Konzepts in den Internationalen Beziehungen

Dieser Beitrag bietet einen Überblick zu zwischenstaatlichem Vertrauen als theoretischem und empirischem Konzept in den IB. Hierzu kontrastieren wir zunächst informationsbasiertes Vertrauen mit einem Vertrauensverständnis, das bei der Aufhebung von Ungewissheit ansetzt. Wir unterstreichen den ontologischen Mehrwert der aufhebungsbasierten Variante, da sich Vertrauen nur aus dieser Perspektive als eigenständiges und abgrenzbares Phänomen verstehen lässt. Anschließend zeigen wir, dass informationsbasiertes Vertrauen auch in den IB wenig Neues mit sich bringt, während aufhebungsbasiertes Vertrauen Innovationspotential besitzt. Allerdings ist aufhebungsbasiertes Vertrauen ein bislang untertheoretisiertes und empirisch nur wenig analysiertes Phänomen. Da Letzteres auch auf fehlende Vertrauensindikatoren zurückzuführen ist, diskutieren wir im letzten Teil des Artikels akteurstheoretische Zugänge zu Vertrauen zwischen Staaten und skizzieren eine mögliche Operationalisierung von zwischenstaatlichem Vertrauen.

\section{Einleitung}

Komplexität und Ungewissheit prägen internationale Politik (Barnett/Adler 1998: 414; Booth/Wheeler 2008: Kap. 1). ${ }^{1}$ Weil sich keine objektiven Erwartungen hinsichtlich des Verhaltens von Staaten bilden lassen, müssen Regierungen immer mit Überraschungen rechnen. Damit sie unter diesen Bedingungen kooperieren, sind Mechanismen notwendig, die Komplexität und Ungewissheit reduzieren. Vertrauen gilt neben Kontrolle als ein solcher Mechanismus. Während Kontrolle darauf abzielt, die Menge unerwünschter Handlungsoptionen künstlich zu beschränken, setzt Vertrauen auf die Integrität und Zuverlässigkeit von Kooperationspartnern. Kontrolle geschieht durch die Manipulation von Umweltbedingungen und Nutzenfunktionen, so dass Akteure ${ }^{2}$ unter den gegebenen Bedingungen starke Gründe haben, sich auf wechselseitige Kooperation einzulassen. Vertrauen ist demgegenüber etwas qualitativ anderes. Es steht und fällt mit der Erwartung, dass sich Kooperati-

1 Dieser Beitrag entstand im Rahmen eines durch die DFG finanzierten Forschungsprojekts zur Konzeptualisierung von Vertrauen in den Internationalen Beziehungen. Für kritische Rückmeldungen und anregende Kommentare $\mathrm{zu}$ früheren Textversionen bedanken wir uns herzlich bei unseren Tübinger Kollegen Thomas Diez, Max Mutschler und Thomas Nielebock, beim Tübinger Institutskolloquium und bei den drei anonymen GutacherInnen der ZIB.

2 Aus Gründen der besseren Lesbarkeit wird durchgängig das generische Maskulinum verwendet, welches männliche und weibliche Personen einschließt. 
onspartner auch dann an ihre Zusagen halten, wenn sie Ausbeutungschancen gefahrlos nutzen könnten.

Mit Ken Booth und Nicholas J. Wheeler (2008: 252) meinen wir, dass Vertrauen in den IB ein stark vernachlässigter, aber höchst lohnenswerter Untersuchungsgegenstand ist. Denn mit fortschreitender Globalisierung nimmt der Bedarf an zwischenstaatlicher Kooperation permanent zu. Wie die Wirtschafts- und Organisationssoziologie zeigt, kann Vertrauen in einem so hoch dynamischen Umfeld zu einem komparativen Vorteil werden. Vertrauen gilt als Schmiermittel sozialer Interaktionen. In der Form von Sozialkapital ermöglicht es robuste Absprachen, fördert offene Kommunikation, erhöht den Glauben in externe Informationen und schafft Raum für gemeinsames soziales Lernen. Darüber hinaus erzeugt Vertrauen ein Gefühl individueller Sicherheit (Bijlsma-Frankema/Costa 2005: 263). Zwar ist bekannt, dass Kontrollmechanismen ähnlich wirken, und zudem lässt sich kaum bestreiten, dass mit der Technologisierung moderner Industriegesellschaften auch deren Überwachungsfähigkeiten gestiegen sind. Ob diese die zunehmende Komplexität effektiv bearbeiten können, darf allerdings bezweifelt werden. Schließlich ist hierfür die Spezifikation und Kodifizierung zulässiger Handlungen, effektives Monitoring und konsequente Sanktionierung notwendig. Es ist jedoch eine Binsenweisheit, dass diese Bedingungen in der Staatenwelt nur schwer herzustellen sind und mit erheblichen Kosten einhergehen. Kontrollbasierte Ansätze der zwischenstaatlichen Kooperation stoßen somit hinsichtlich ihrer Effektivität und Effizienz schnell an ihre Grenzen. Ein Mehr an Vertrauen ist deshalb wünschenswert: denn »Vertrauen ist gut, Kontrolle ist teuer« (Fiedler 2001: 576).

In der einschlägigen Literatur finden wir starke theoretische Argumente und erste empirische Hinweise auf die Wirksamkeit von Vertrauen in der internationalen Politik. Es fehlen uns allerdings noch die passende analytische Brille sowie die notwendigen Instrumente, um Vertrauen als solches sichtbar zu machen und bei Bedarf in verantwortbarer Weise zu stärken. Dieser Literaturüberblick möchte deshalb über unterschiedliche Vertrauenskonzeptionen, über den aktuellen Forschungsstand in den IB und über mögliche empirische Zugänge zu zwischenstaatlichem Vertrauen informieren. Hierzu greifen wir in unserem Beitrag auf ein breites Spektrum an Literatur aus der Philosophie, der Soziologie, der Organisationssoziologie, den Betriebswissenschaften und freilich den Politikwissenschaften selbst zurück.

Nach einer ersten formalen Klärung des Vertrauensbegriffs setzen wir uns im dritten Abschnitt mit der Vertrauensontologie rationalistischer Ansätze auseinander. Auch wenn ein Großteil der allgemeineren Literatur Vertrauen aus dieser metatheoretischen Perspektive thematisiert, sieht sich die rationalistische Vertrauensontologie substantieller Kritik ausgesetzt. So wird zum einen argumentiert, dass sich Vertrauen im Sinne eines informationsbasierten Kalküls kaum noch von Kontrolle unterscheiden lässt. Zum anderen wird bezweifelt, dass Vertrauen - wenn es denn mehr ist als informationsbasiertes Kalkül - überhaupt rationalen Ursprungs sein 
kann. ${ }^{3}$ Wie wir im vierten Abschnitt ausführen werden, beanspruchen nicht-rationalistische Ansätze dem Vertrauensphänomen besser gerecht werden zu können. Sie betonen die Aufhebung von Ungewissheit und unterscheiden strikt zwischen Vertrauen und Kontrolle. Wir halten die Kritik am rationalistischen Vertrauensverständnis für berechtigt. Entsprechend zeigen wir in Auseinandersetzung mit der einschlägigen Literatur, dass dem als Aufhebung begriffenen Vertrauen nicht nur ontologische Eigenständigkeit zugesprochen werden kann, sondern dass ihm ein extra-rationales Moment innewohnt, welches es für rationalistische Modelle schwer zugänglich macht.

Im fünften Abschnitt fassen wir den einschlägigen Forschungsstand zu Vertrauen und Vertrauensbildung in den IB zusammen. Dabei konzentrieren wir uns vornehmlich auf die Themenbereiche der Friedens- und Konfliktforschung. Es wird sichtbar, dass rationalistische Perspektiven zwar vergleichsweise weitverbreitet sind, aber wenig innovatives Potential besitzen. Demgegenüber zeigen Arbeiten zur Bildung internationaler Identitätsgemeinschaften, zur Außenpolitikanalyse, zu Versöhnung und zu Respekt und Freundschaft zwischen Staaten, dass die alternative, aufhebungsbasierte Konzeption von Vertrauen als eigenständiges Phänomen durchaus in den IB angekommen ist. Allerdings fehlen noch die theoretischen wie empirischen Instrumente, um seine faktische Relevanz angemessen diskutieren zu können.

In den beiden letzten Teilen unseres Forschungsüberblicks gehen wir deshalb auf zwei zentrale Herausforderungen der aktuellen Vertrauensforschung ein. Zum einen diskutieren wir die Frage der akteurstheoretischen Verortung von Vertrauen in den IB. Hier geht es um die Frage, wer wem vertraut, wenn wir von Vertrauen zwischen Staaten sprechen. Zum anderen skizzieren wir einen Versuch, Vertrauen in der internationalen Politik sichtbar zu machen. Ohne valide Messverfahren kann selbst ein hochplausibles Konzept für die Forschung kaum analytischen Mehrwert erbringen. Theoretische Konstrukte müssen empirisch zugänglich sein, wenn sie helfen sollen, internationale Politik besser zu verstehen und zu erklären.

3 Wir bewerten rationalistische Vertrauenskonzepte unter Verwendung eines anspruchsvollen Rationalitätsbegriffs. Wir gehen davon aus, dass die Rationalität der Akteure common knowledge darstellt: Der Vertrauensgeber weiß, dass der Vertrauensnehmer kalkülbasiert handelt und deshalb seine kooperativen Präferenzen umweltabhängig variieren werden. Ebenso gehen wir von einem strengen Konzept subjektiver Wahrscheinlichkeiten aus: Die Wahrscheinlichkeit als subjektiver degree of belief muss auf Informationen und/oder empirischen Erfahrungen basieren (im Sinne eines rational belief), und soll nicht als bloße psychologische Variable verstanden werden. An dieser Stelle wollen wir auch klarstellen, dass wir rationalistische Ansätze nicht allgemein in ihrem normativen Charakter oder ihrer empirisch bewährten Prognose- und Erklärungsfähigkeit hinterfragen. Vielmehr kritisieren wir ihren deskriptiven bzw. ontologischen Anspruch in der konkreten Vertrauensforschung. Wir meinen, dass die rationalistische Konzeptualisierung von Vertrauen zu einer verminderten Erklärungs- und Prognosefähigkeit über Ursachen und Wirkungen von Vertrauen in sozialen Kontexten führen kann. 


\section{Vertrauen allgemein}

Vertrauen teilt das Schicksal vieler Kernkonzepte in den Sozialwissenschaften: Ihm fehlt eine allgemein akzeptierte Definition. Gleichwohl herrscht in der einschlägigen Literatur ein bemerkenswert breiter Konsens zu einer Reihe ontologischer Grundmerkmale dessen, was Vertrauen genannt werden kann (Coleman 1994; Deutsch 1962; Hardin 2006; Luhmann 2009). So wird Vertrauen auf Kooperationsprobleme in strategischen Settings bezogen: Ein Akteur, der Vertrauensgeber, entscheidet sich für oder gegen eine Vertrauenshandlung in Form einer kooperativen und riskanten Vorleistung gegenüber dem Vertrauensnehmer in Abhängigkeit von dessen zu erwartender Reaktion (Vertrauenserwartung) (Ripperger 1998: 42-46). Die Vertrauenserwartung beschreibt somit die Erwartung vertrauenswürdigen Verhaltens in Form erwiderter Kooperation durch den potentiellen Vertrauensnehmer. Sie zielt dabei eindeutig auf die Motivation des Vertrauensnehmers zu vertrauenswürdiger Reaktion, und nicht auf seine Kompetenz. ${ }^{4}$

Das Vertrauensproblem ergibt sich aus der zeitlichen Verzögerung (Reaktionszeit) zwischen einer Vorleistung zum Zeitpunkt $t_{0}$ und der möglichen Erwiderung durch den Nehmer zum Zeitpunkt $t_{1}$ (siehe Abbildung 1) (Barbalet 1996: 77-79, 81-84; Luhmann 2009: 8; Möllering 2006b: 105-126). Die soziale Realität ist hochgradig komplex und die Zukunft generell unsicher. Wenn überhaupt, ist sie nur sehr beschränkt prognostizierbar. Folglich kann der Vertrauensgeber die Reaktion seines Kooperationspartners nicht sicher vorhersagen. Aufgrund der inhärenten Unsicherheit ${ }^{5}$ und des Schadens, der sich aus enttäuschtem Vertrauens ergibt, ist Vertrauen immer ein Wagnis. ${ }^{6}$ Eine positive Vertrauenserwartung jedoch ermöglicht es den Akteuren, dieses Wagnis einzugehen. Denn Vertrauen funktioniert im Sinne von Niklas Luhmann als »Mechanismus der Reduktion sozialer Komplexität« (Luhmann 2009). Es bewirkt auf diesem Weg die Festigung einer Erwartung kooperativen Verhaltens durch den Vertrauensnehmer.

4 Wenige Ansätze beziehen die Vertrauenserwartung auch auf die Kompetenz des Interaktionspartners (Das/Teng 2001: 256, 257; 2004: 97; Yamagishi/Yamagishi 1994: 131).

5 Booth/Wheeler (2008: 40) betonen, dass sich Unsicherheit im Rahmen des Vertrauensproblems auf die zukünftigen, und nicht auf aktuelle Handlungsabsichten des Interaktionspartners bezieht (future uncertainty).

6 Wir vermeiden ausdrücklich die Verwendung des Risiko-Begriffs aufgrund seiner Bedeutung in der rationalistischen Theorie. 


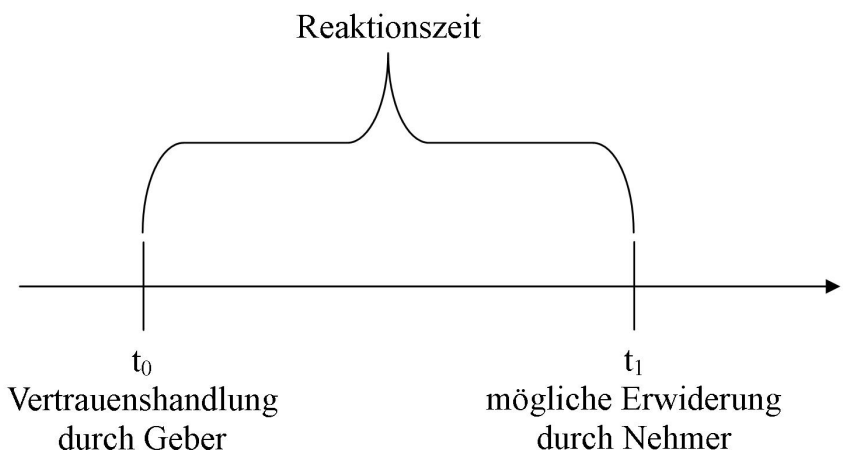

Vertrauen ist von zwei anderen komplexitätsreduzierenden Mechanismen abzugrenzen: Misstrauen und Kontrolle. Misstrauen ist das Gegenteil von Vertrauen: Es erzeugt negative Erwartungen vertrauensunwürdigen Verhaltens. Dabei wird diese Erwartung auf die inhärent unkooperativen Präferenzen des Interaktionspartners zurückgeführt. Wir begreifen starkes Misstrauen und starkes Vertrauen als zwei Enden eines Kontinuums, dessen Zentrum am besten durch »Neutralität« beschrieben werden kann: Wenn ein Akteur seinen Interaktionspartner (noch) nicht kennt, hat er sich schlichtweg (noch) keine Meinung über seine Zuverlässigkeit gebildet und kann ihm somit weder vertrauen noch misstrauen. Demensprechend ist die Komplexität der Entscheidungssituation im Neutralitätszustand maximal, während Misstrauen und Vertrauen sie reduzieren und (starke) Erwartungen erlauben.

So wie Vertrauen dient auch Kontrolle der Bildung positiver Kooperationserwartungen: Während sich allerdings Vertrauen auf die Einschätzung eines Akteurs als vertrauenswürdig bezieht, basieren Erwartungen im Fall von Kontrolle auf dem Wissen über die kooperationsfördernde Wirkung struktureller Rahmenbedingungen (Möllering 2005: 287-288). Unter das Konzept solcher Kontrollstrukturen kann eine Reihe bekannter Konzepte aus der Kooperationstheorie subsumiert werden, wie etwa (Inter-)dependenz, Reputationssorgen, Abschreckung, Sanktionen und Hostage-Taking. Uns ist wichtig, auch Überwachung in Form kontinuierlicher Informationseinholung und -verarbeitung als Form der Kontrolle zu begreifen (siehe auch Blum 2003: 365-369): Denn wer nicht genug vertraut, wird sich ständig rückversichern müssen, um im Fall antizipierten, unkooperativen Verhaltens präventive Gegenmaßnahmen ergreifen zu können, die solches Verhalten unrentabel werden lassen.

Mit Hilfe dieser sehr allgemein gehaltenen Annäherung an den Vertrauensbegriff lassen sich unterschiedliche in der Literatur etablierte Vertrauenskonstellationen erfassen (Uslaner 2002: 26-29): Bringt Akteur A eine positive Vertrauenserwartung nicht näher spezifizierten Akteuren entgegen, so handelt es sich um generalisiertes Vertrauen bzw. eine generalisierte Vertrauenserwartung. Vertraut A hingegen 
einem konkreten Akteur B, haben wir es mit spezifischem Vertrauen, bzw. einer spezifischen Vertrauenserwartung zu tun. Spezifisches Vertrauen kann dabei sowohl eine Interaktionsbeziehung als solche kennzeichnen (A vertraut B) als auch eine Interaktionsbeziehung mit Bezug auf ein konkretes Objekt $\mathrm{X}$ der Interaktion (A vertraut B in Bezug auf $\mathrm{X}$ ).

Nach welcher Logik Vertrauen im Sinne der Bereitschaft zu riskanten Vorleistungen funktioniert, ist umstritten. Dabei verläuft eine der zentralen Konfliktlinien innerhalb der Vertrauensliteratur zwischen rationalistischen und nicht-rationalistischen Ansätzen, die wir so übernehmen und auf die wir uns im Folgenden konzentrieren wollen. In diesem Zusammenhang setzen wir uns hauptsächlich mit Konzepten spezifischen Vertrauens auseinander und streifen das Phänomen generalisierten Vertrauens nur am Rande.

\section{Vertrauen als Kalkül}

\subsection{Vertrauen als Kalkül in den Sozialwissenschaften}

Aus rationalistischer Perspektive erscheint Vertrauen als subjektives Kalkül, das der Vertrauensgeber auf der Basis von Erfahrungen und aktuellen Informationen bildet (Hardin 2002: Kap. 1; Ripperger 1998: Kap. 4). Dies setzt voraus, dass der Vertrauensgeber zukünftige Handlungspräferenzen seines Kooperationspartners hinreichend präzise abschätzen kann, um die Vertrauenserwartung in Form einer subjektiven Wahrscheinlichkeit p zu bilden. Sie gleicht dem degree of belief des Vertrauensgebers über vertrauenswürdiges Handeln des Nehmers. Gemäß der gängigen rationalistischen Entscheidungstheorie erbringen Akteure dann riskante Vorleistungen, wenn ihr Erwartungsnutzen positiv ist. ${ }^{7}$ Dementsprechend kann Vertrauen als Entscheidung unter Unsicherheit modelliert werden. ${ }^{8}$

Das Vertrauensproblem kann nach rationalistischer Überzeugung als typisches Informationsproblem durch Bayessche Lernprozesse gelöst werden (Hardin 2002: 113; Kydd 2000: 333). Dabei gewinnt der Vertrauensgeber auf Basis von Informationseinholung und -verarbeitung fortlaufend Erkenntnis über die Präferenzen des Vertrauensnehmers. Die subjektive Wahrscheinlichkeit p wird dann im Lichte dieser Erkenntnisse ständig neu justiert. Vergangene Erfahrungen werden zur Voraussetzung gegenwärtiger Erwartungen und bilden die Grundlage für mehr oder weniger starkes Vertrauen in die zukünftigen Handlungsabsichten des Kooperationspart-

7 Teilweise wird für die Hinzunahme der Bedingung von $\mathrm{p}>0.5$ plädiert, um Vertrauensentscheidungen von purem Gambling unterscheiden zu können (Deutsch 1958; 1962; Ripperger 1998: 88-90).

8 Bei Entscheidungen unter Ungewissheit (ignorance/hard/fundamtental uncertainty) sind mögliche Zukunftsszenarien und Eintrittswahrscheinlichkeiten nicht antizipier- und kalkulierbar. Unter Unsicherheit (uncertainty) können Wahrscheinlichkeiten subjektiv geschätzt werden und unter Risiko (risk) sind objektive Wahrscheinlichkeiten gegeben. Unter Sicherheit ist die Zukunft eindeutig vorhersagbar (Davidson 1991: 130, 131; Morrow 1994: 28, 29). 
ners. Die Bildung der Vertrauenserwartung gleicht somit der Formulierung einer probabilistischen Theorie über die Vertrauenswürdigkeit des Vertrauensnehmers. Dabei macht die Möglichkeit umweltabhängig variierender Präferenzen seitens des Nehmers eine solche Theorieformulierung zu einem komplexen Unterfangen. Wie Tanja Ripperger (1998: 114-115) zeigt, sieht sich der Geber zweier Unsicherheitsquellen gegenüber und bildet die Wahrscheinlichkeit $\mathrm{p}$ als Aggregat aus mehreren subjektiven Wahrscheinlichkeitsverteilungen (siehe Abbildung 2): zum einen aus den Eintrittswahrscheinlichkeiten möglicher Umweltzustände $p\left(U_{n}\right)$, zum anderen aus den Eintrittswahrscheinlichkeiten vertrauenswürdiger Reaktionen unter den jeweils möglichen Umweltzuständen $\mathrm{p}\left(\mathrm{K} \mid \mathrm{U}_{\mathrm{n}}\right)$.

Abbildung 2: Die Bildung der Vertrauenserwartung gemäß rationalistischer Modellannahmen

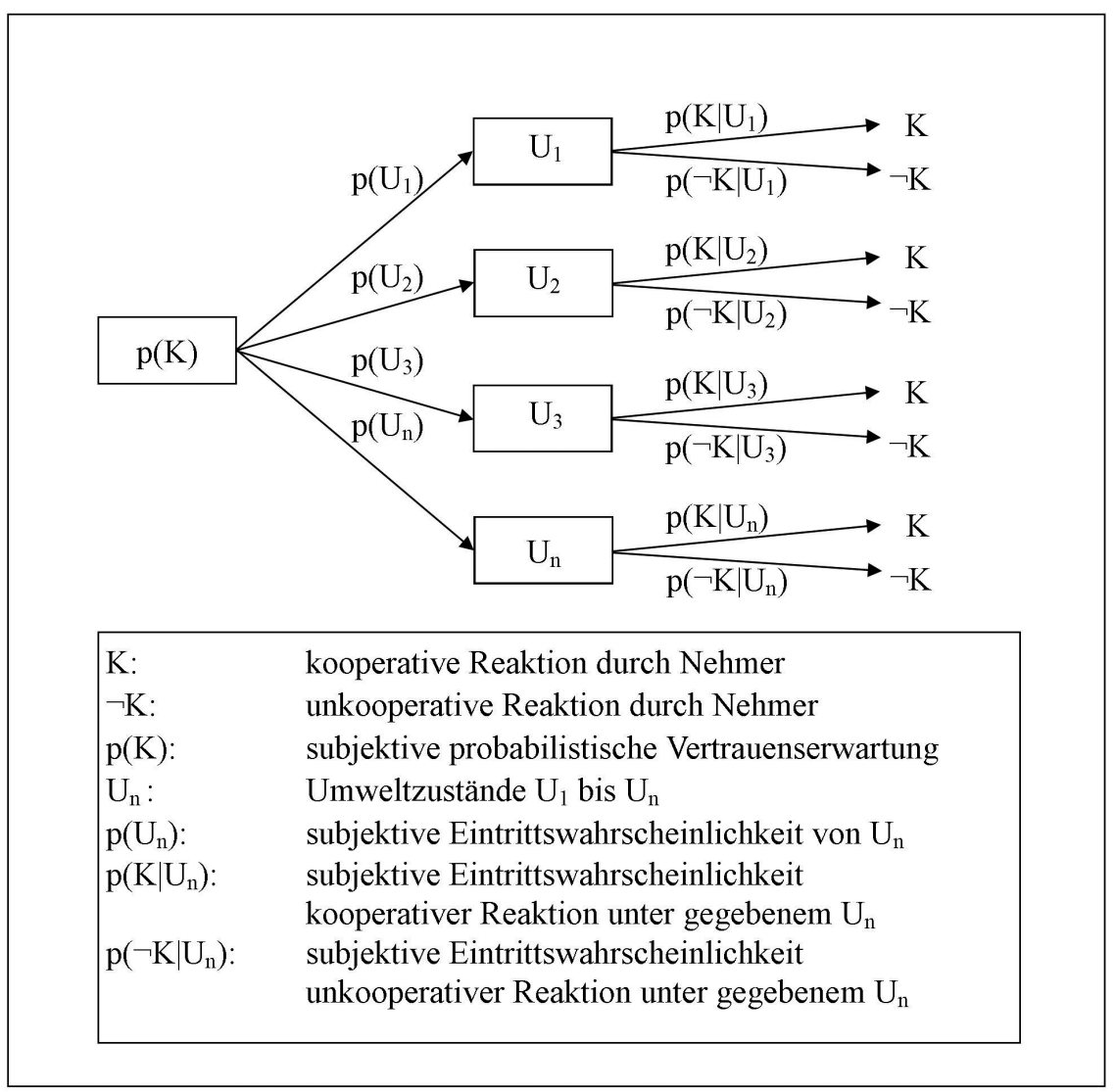


Seitens des Vertrauensnehmers ist in der rationalistischen Vertrauensontologie vertrauenswürdiges Verhalten immer dann begründet, wenn die Ausbeutung der Vorleistung soweit erkennbar nicht im wohlverstandenen Eigeninteresse des Vertrauensnehmers liegt. Oder anders formuliert: Das Interesse des Vertrauensgebers muss mit hinreichend großer Wahrscheinlichkeit im Interesse des Vertrauensnehmers eingeschlossen sein. Russell Hardin (2002: 3) nennt dies auch »encapsulated interest $\ll$. Dieser Zugang erlaubt es, unterschiedliche Motive für vertrauenswürdiges Verhalten zu benennen. Eine Situation von Interessenverschränkung besteht etwa, wenn dem Vertrauensnehmer aufgrund eines langen Schattens der Zukunft an einer Aufrechterhaltung kooperativer Beziehung gelegen ist, wenn Reputationssorgen Akteure zu kooperativem Verhalten bewegen, oder aber wenn drohende Sanktionen kooperative Reaktionen hervorrufen. Bezüglich der Rolle von moralischen und altruistischen Präferenzen, wie sie teilweise auch in rationalistischen Modellen modelliert werden (Braun 1992: 180-184), argumentiert Hardin, dass diese zwar auch unter den »encapsulated interest«-Begriff subsumiert werden können, die realweltliche Relevanz solcher Präferenzen vor dem Hintergrund des dem rationalistischen Paradigma zugrunde liegenden Menschenbildes jedoch eher marginal zu sein scheint (Hardin 2002: Kap. 2).

\subsection{Kritik am rationalistischen Konzept}

Auch wenn die rationalistische Vertrauensontologie die einschlägige Literatur bislang dominiert, ist sie nicht unumstritten. Ein genereller Kritikpunkt lautet, dass sie Vertrauensverhältnisse auf Interessenkonvergenz zwischen Egoisten reduziere, Vertrauen aber eine andere Qualität von Beziehung bezeichne. Etwas spezifischer formuliert beanstanden Kritiker die Konfusion von Vertrauen und Kontrolle und rekurrieren dabei auf drei Argumente: Erstens ist die ontologische Überschneidung von Vertrauen und Kontrolle für jene Vertrauenskonzepte offensichtlich, in denen die Wirkung handlungseinschränkender Abschreckungs- oder Sanktionsmechanismen als Ursache von Vertrauenserwartungen begriffen werden (siehe bspw. deterrence-based trust (Rousseau et al. 1998: 398)). Zweitens ist eine solche Überschneidung evident in Modellen, die auf der Annahme allgemeingültiger und fixer Präferenzen basieren. Die Reaktion des Nehmers wird hier maßgeblich durch die situationsspezifische Struktur beeinflusst. Deshalb bedarf es zur Bildung einer positiven Vertrauenserwartung lediglich der Antizipation einer kooperationsfreundlichen Spielstruktur. Vertrauen wird zur bloßen »Situationsvariable« (Lahno 2001: 313): Sobald sich die kooperationsfördernde Situationsstruktur auflöst, wird dem Vertrauen die Grundlage entzogen. Akteursspezifische Eigenschaften spielen allenfalls eine marginale Rolle. Drittens gehen Kontrolle und Vertrauen in der rationalistischen Ontologie ineinander über, weil ein Vertrauensnehmer unter realweltlichen Bedingungen nicht von Unsicherheit und fixen Präferenzen beim Vertrauensneh- 
mer ausgehen kann. Dies entspricht einem Standardeinwand gegen einfache, rationalistische Modelle:

»As soon as we seek to predict the behavior of other human beings, we should begin to be suspicious of discussions that take the Bayesian decision theory for granted. After all, if Bob is as complex as Alice, it is impossible for her to create a model of the world that incorporates a model of Bob that is adequate for all purposes « (Binmore 2009: 146).

Vielmehr findet Vertrauen unter Ungewissheit statt (Möllering 2006b: 8). ${ }^{9}$ Denn der Raum möglicher Umweltzustände und damit einhergehender Nehmerreaktionen ist letztendlich auf der Grundlage des verfügbaren Wissens nicht kalkulierbar. Immer wieder können unvorhergesehene Situationen zu neuen Interessenkonstellationen führen und Kooperation fraglich werden lassen. Folglich lässt sich zukünftiges Verhalten auf der Basis selektiver Erfahrungen nicht sinnvoll prognostizieren. ${ }^{10}$ Darüber hinaus können sich Nehmerpräferenzen über Zeit hinweg verändern. Nach John J. Mearsheimer gilt beispielsweise, dass »a state's intention can be benign one day and hostile the next« (Mearsheimer 2001: 31). Auch deshalb reichen noch so detaillierte Informationen über vergangenes und aktuelles Verhalten nicht aus, um zukünftiges Handeln zu prognostizieren: »Knowledge of a state's present motivation, while valuable, will not suffice to perfectly predict future behavior « (Kydd 2005: 202). Aufgrund der räumlichen und zeitlichen Kontingenzen wird das dem Vertrauen zugrunde liegende Informationsproblem somit zum Humeschen Inferenzproblem, welches durch die bloße Ansammlung von Informationen nicht gelöst werden kann, da der Vertrauensgeber sich unabwendbar mit dem Problem von unkown unknowns konfrontiert sieht. Dem rationalistisch Vertrauenden ist es schlichtweg nicht möglich, stabile Erwartungen zu bilden: Vielmehr muss er seinen Interaktionspartner fortwährend überwachen, um unkooperatives Verhalten zu antizipieren und präventiv einzuschreiten. $\mathrm{Zu}$ dieser Schlussfolgerung kommt auch Andrew H. Kydd: Im Fall variabler Präferenzen könne eine Vertrauenserwartung nur durch fortwährende Informationseinholung und -verarbeitung gebildet und aufrecht erhalten werden (Kydd 2005: 202-203). Für Kydd stellt ein solches Verhalten zwar kein Problem dar, da es im Einklang mit seinem Vertrauensverständnis steht. In der Literatur wird es jedoch mehrheitlich als Kontrollverhalten und als Gegensatz zu vertrauensvollem Verhalten interpretiert (Blum 2003: 365-369). ${ }^{11}$

Insgesamt stimmen wir mit der rationalismuskritischen Literatur darin überein, dass eine vom Kontrollmechanismus klar unterscheidbare Vertrauenserwartung auf Basis rationalistischer Modellannahmen weder konzeptualisierbar noch erklärbar ist. Dies gilt vor allem, wenn man akzeptiert, dass das eigentliche Vertrauensproblem durch einen Zustand unabwendbarer Ungewissheit gekennzeichnet ist. Demensprechend muss gefragt werden, ob das Vertrauensphänomen in seiner Gesamt-

9 Booth und Wheeler $(2008: 4,23)$ sprechen auch von »unresolvable uncertainty«.

10 Eine solche Verallgemeinerung steht auch im Kontrast du den Modellannahmen einer anspruchsvollen rationalistischen Theorie (Davidson 1991).

11 Kydd selbst entgeht dem Problem der konzeptionellen Überschneidung von Kontrolle und Vertrauen, indem er von stabilen Präferenzen ausgeht - eine für uns zu einfache und realweltlich nicht plausible Annahme. 
heit durch eine rationalistische Ontologie überhaupt erfasst werden kann. ${ }^{12}$ Dies scheint allenfalls dann möglich zu sein, wenn Wahrscheinlichkeitsschätzungen als rein psychologische Disposition begriffen und an den Prozess der Erwartungsbildung keine strengeren Modellanforderungen gestellt werden. Wir meinen, dass die Bildung der Vertrauenserwartung dann allerdings nicht mehr einer rational belief formation gleicht, weshalb wir auch eher von einer psychologisierten Spielart des Rationalismus sprechen möchten. ${ }^{13}$ Generell sollte jedenfalls deutlich geworden sein, dass eine strenge rationalistische Perspektive es nicht schafft, den Kern des Vertrauensphänomens zu explizieren. Wenn Vertrauen und Kontrolle nicht mehr trennbar sind und Vertrauen auf einen komplexen Prozess der kontinuierlichen Informationseinholung und -verarbeitung angewiesen ist, dann dient Vertrauen nicht der Luhmannschen Reduktion von Komplexität. Vielmehr würde versucht, der Komplexität der sozialen Welt durch ihre mentale Ordnung und Strukturierung Herr zu werden. Wir meinen aber, dass Vertrauen erst dann relevant wird, wenn es um die Reduktion einer unüberschaubaren Welt geht, die sich mental überhaupt nicht angemessen ordnen und strukturieren lässt. Nach den Worten von Hansjörg Siegenthaler (1993: 92) wird Vertrauen notwendig, da »gegen die residuale Unsicherheit ${ }^{14}[\ldots]$ kein Informationskraut gewachsen« ist.

\section{Vertrauen als Aufhebung von Ungewissheit}

\subsection{Vertrauen als Aufhebung in den Sozialwissenschaften}

Ein grundlegendes Merkmal der rationalismuskritischen Literatur ist es, dass durch die Betonung extra-rationalistischer Grundannahmen Vertrauen strikt von Kontrolle abgegrenzt wird. Allerdings wird auch von den Vertretern und Vertreterinnen des alternativen Konzepts eine Superrationalität der Akteure unterstellt: Der Vertrauensgeber nimmt nur dann eine Vertrauenshandlung vor, wenn er eine vertrauenswürdige Reaktion erwarten kann. Gleichzeitig wird aber die extra-rationale Konzeption der Vertrauenserwartung selbst betont. Während im rationalistischen Modell Eigeninteresse und ein informationsbasiertes Kalkül den konzeptionellen Kern

12 Inwieweit komplexere rationalistische Modelle existieren, die eine Modellierung stabiler und wenig informationsabhängiger Erwartungen unter Ungewissheit erlauben, lassen wir bewusst offen. Fakt ist, dass solche Modelle in der vorliegenden Vertrauensliteratur nicht benannt werden. Vielmehr wird das oben skizzierte rationalistische Modell mit alternativen Konzepten kontrastiert, die den vermeintlich extra-rationalen Kern des Vertrauensphänomens herausstellen und Vertrauen eindeutig von Kontrolle unterscheidbar machen.

13 Auch Leonard Savage (1954: 15-20), Mitbegründer der subjektiven Wahrscheinlichkeitstheorie, argumentiert, dass seine Theorie hauptsächlich der Deskription von Entscheidungen unter Unsicherheit in small worlds dienen kann. In large worlds, also unter Ungewissheit wäre dies nur eingeschränkt möglich. Tatsächlich existiert eine lebhafte Diskussion zu dieser Thematik, weshalb wir uns eine endgültige Meinung nicht anmaßen wollen (exemplarisch Davidson 1991; Gilboa et al. 2012). Siehe auch Jens Beckerts (1996) Plädoyer für eine stärkere Beschäftigung mit dem Konzept der Ungewissheit.

14 In unserer Terminologie meint Siegenthaler damit Ungewissheit. 
ausmachen, werden jetzt die (moralische) Integrität und Zuverlässigkeit der Akteure und die Aufhebung von Ungewissheit als zentrale Besonderheiten von Vertrauen markiert (Tabelle 1 fasst die Kritik am rationalistischen Modell zusammen und grenzt sie von Vertrauen im Sinne der Aufhebung von Ungewissheit ab). ${ }^{15}$

Gemäß der ersten Besonderheit gründet die Vertrauenserwartung des Vertrauensgebers ausschließlich in akteursspezifischen Eigenschaften des Vertrauensnehmers. In diesem Sinne erwartet der Geber, dass der Nehmer sich nicht aus egoistischen Eigeninteressen heraus, sondern aufgrund seiner moralischen Integrität als zuverlässig erweisen wird, und zwar konstant und weitgehend ${ }^{16}$ unempfindlich gegenüber äußeren Umständen. ${ }^{17}$ Dementsprechend sieht der Geber den vertrauenswürdigen Nehmer in einem »normatively positive light as >upright $<$, >honorable $<$, >truthful«, >loyal and 〉scrupulous«« (Hoffman 2002: 381). Der Nehmer wird als sozial eingebetteter Akteur wahrgenommen, der eine starke Disposition zur Regeleinhaltung hat und in seinem Handeln denselben sozialen Normen folgt wie der Vertrauensgeber. ${ }^{18}$ Diese Erwartung ist für einen vertrauenden Akteur primär handlungsleitend: Selbst wenn strukturelle Gegebenheiten den Nehmer ohnehin zu kooperativem Handeln bewegen würden, wird dessen kooperatives Verhalten letztlich auf seinen Charakter zurückgeführt. Diese Modellannahmen werden durch experimentelle Studien unterstützt. Sie zeigen, dass für eine Kooperationsentscheidung des Vertrauensgebers weniger die objektiv gegebenen Pay-Off-Strukturen ausschlaggebend sind als die subjektiv zugeschrieben Persönlichkeitsmerkmale des potentiellen Kooperationspartners. ${ }^{19}$ Offenkundig rechnen viele Vertrauensgeber mit einer stabilen persönlichen Disposition des Vertrauensnehmers, die ihn auch dann noch kooperieren lässt, wenn es sich für ihn streng genommen nicht mehr lohnt.

Nach Maßgabe der zweiten Besonderheit erlaubt es Vertrauen gerade unter Ungewissheit und unabhängig von fortwährendem Informationsupdating stabile Erwartungen über die Reaktionen des Interaktionspartners zu bilden. Obwohl diese Erwartungen nicht überprüfbar sind, sind sie beim Vertrauensgeber hinreichend stark ausgeprägt, um ihn zu kooperativem Handeln zu bewegen »als ob« ihr Wahrheitsgehalt sicher sei (Messick/Kramer 2003: 91). Demensprechend bezeichnet Georg Simmel (1983: 263) eine Vertrauenserwartung auch als »Zustand zwischen Wissen und Nichtwissen«. Nach Guido Möllering (2006a: 4) ist es die Fähigkeit zur Aufhebung oder auch Ausblendung von Ungewissheit (Suspension), die dazu befähigt, eine Welt zu imaginieren, in der die vertrauenswürdige Reaktion des Part-

15 Natürlich existiert nicht das eine alternative Vertrauenskonzept. Existierende Konzepte betonen die von uns diskutierten Eigenschaften unterschiedlich stark.

16 Die Qualifizierung »weitgehend « ist an dieser Stelle wichtig, da es auch aus moralischer Sicht Zumutbarkeitsgrenzen gibt. Hierzu zählt beispielsweise, wenn der Kooperationspartner von seiner Seite her Vereinbarungen bricht.

17 Ripperger (1998: 116-117) formalisiert die Idee umweltunabhängiger Vertrauenswürdigkeit. Allerdings handelt der Nehmer immer noch nutzenorientiert, integres Handeln spielt keine Rolle.

18 Dieser Gedanke wurde auch in den Arbeiten von Morton Deutsch (1958) erörtert.

19 Siehe Cook et al. (2005); Hayashi et al. (1999); Kuhlman/Marshello (1975); McClintock/ Liebrand (1988); Preisendörfer (1995); Rotter (1967;1980); Yamagishi et al. (2005); Yamagishi et al. (1999). Für einen Überblick siehe auch Cook/Cooper (2003). 
ners nicht in Frage steht. ${ }^{20}$ Aufhebung oder Ausblendung klammert die Möglichkeit des Vertrauensbruchs schlichtweg aus und transformiert objektiv bestehende Ungewissheit in subjektive Sicherheit. ${ }^{21}$ Sie erlaubt die unaufwändige Bearbeitung räumlicher und zeitlicher Kontingenzen, indem sie zwei Überzeugungen stabilisiert: Zum einen, dass auch in unvorhersehbaren Situationen, also »komme was wolle«, der Nehmer integres Verhalten an den Tag legt; zum anderen, dass der Nehmer sich in seinem vertrauenswürdigen Charakter auch über die Zeit hinweg nicht verändern wird. ${ }^{22}$ Aufhebung oder Ausblendung ermöglicht auf diesem Wege die Bildung stabiler Erwartungen, ohne dabei auf fortwährende Informationseinholung angewiesen zu sein, denn Vertrauen impliziert »confidence concerning one's own judgment of another« (Barbalet 2009: 375). Dementsprechend wird das dem Vertrauen zugrunde liegende Informationsproblem schlichtweg aufgehoben. Deshalb kommt Vertrauen tatsächlich dem oft zitierten Glaubenssprung - einem »leap of faith « - gleich (Lewis/Weigert 1985; Möllering 2006b: Kap. 5). ${ }^{23}$ Dabei muss davon ausgegangen werden, dass umso stärkere Aufhebungsprozesse notwendig werden, je größer die Reaktionszeit bis zur möglichen Vertrauenserwiderung ist. Schließlich wird Ungewissheit umso größer, je weiter Handlungen in der Zukunft liegen. ${ }^{24}$

Vor dem Hintergrund der beiden diskutierten ontologischen Besonderheiten wird ersichtlich, dass Vertrauen nicht mehr zwischen Egoisten, sondern innerhalb von Wertegemeinschaften entsteht. Zudem werden Vertrauen und Kontrolle nun klar voneinander unterscheidbar: Indem der Vertrauensgeber seine Erwartung auf die moralische Integrität des Nehmers stützt und er zudem auf der Basis von Aufhebungsprozessen auf kontinuierliches Informationsupdating verzichten kann, werden Kontrollmechanismen als Quelle positiver Kooperationserwartungen nebensächlich. Auf diese Weise wird Vertrauen tatsächlich zu einem Mechanismus der Komplexitätsreduktion. Aufwändiges Informieren und Kontrollieren dienen nicht mehr als Basis von Vertrauen, denn nun gilt: »trust begins where prediction ends« (Lewis/Weigert 1985: 976; siehe auch Williamson 1993).

20 Möllering orientiert sich am Hegelschen Begriff der Aufhebung, dem eine Doppelbedeutung innewohnt, da es Negieren und Aufbewahren gleichermaßen mit einschließt (Möllering 2001: 409; 2006a).

21 Bernd Lahno (2002: 43-45) meint, dass der Vertrauende zwar immer noch die Alternative des Vertrauensbruchs wahrnimmt, aber davon ausgeht, dass der Vertrauensnehmer diese Alternative nicht wählen wird.

22 In diesem Sinne können auch jene rationalistischen Modelle, die versuchen, Entscheidungen unter Ungewissheit zu formalisieren (bspw. Binmore 2009: Kap. 9; Kelsey/Quiggin 1992), nicht mit Vertrauen gleichgesetzt werden.

23 Entsprechend meint Möllering (2006b: Kap. 5.4) auch, dass Vertrauen im Letzten ein Willensakt sei und spricht von einem »will to believe« (siehe auch Svendsen 2008: 97-101).

24 Möchte man Vertrauen trotzdem als Wahrscheinlichkeitsschätzung modellieren, so geht dies in Form einer psychologisierten, rationalistischen Vertrauenstheorie. Aufhebungsprozessen könnte dann die Rolle zugesprochen werden, die Generalisierung von selektiven Erfahrungen und somit eine Formulierung einer Wahrscheinlichkeit trotz ungewisser Umweltbedingungen zu ermöglichen. 


\section{Tabelle 1: Zusammenfassung der ontologischen Argumentation}

\begin{tabular}{|c|c|}
\hline Kritik am rationalistischen Modell & Lösung im aufhebungsbasierten Modell \\
\hline $\begin{array}{l}\text { Eine Vertrauensbeziehung ist mehr als eine Inter- } \\
\text { essengemeinschaft von Egoisten. }\end{array}$ & $\begin{array}{l}\text { Der Geber sieht den Nehmer als integre Person } \\
\text { und Teil einer besonderen Beziehung an. }\end{array}$ \\
\hline $\begin{array}{l}\text { Vertrauen findet unter Ungewissheit statt, eine ra- } \\
\text { tionale Bildung der Vertrauenserwartung setzt aber } \\
\text { die Annahme von Unsicherheit voraus. }\end{array}$ & $\begin{array}{l}\text { Das irrationale Moment der Aufhebung ermöglicht } \\
\text { Modellierung von Vertrauen unter Ungewissheit. }\end{array}$ \\
\hline $\begin{array}{l}\text { Vertrauen und Kontrolle sind nicht unterscheidbar, } \\
\text { wenn } \\
\text { 1) die Wirkung struktureller Kontrollen als Quelle } \\
\text { der Vertrauenserwartung dienen darf, } \\
\text { 2) in Modellen mit fixen Präferenzen Vertrauen } \\
\text { auf eine Situationsvariable reduziert wird, } \\
\text { 3) fortwährende Informationseinholung notwendig } \\
\text { für die Bildung und Aufrechterhaltung einer Ver- } \\
\text { trauenserwartung ist. }\end{array}$ & $\begin{array}{l}\text { Vertrauen und Kontrolle werden unterscheidbar, } \\
\text { da } \\
\text { 1) die Erwartung ausschließlich auf die akteurs- } \\
\text { spezifischen Eigenschaften des Nehmers abzielt, } \\
\text { 2) strukturelle Determinanten dabei keine Rolle } \\
\text { spielen, } \\
\text { 3) Aufhebungsprozesse die fortwährende Informa- } \\
\text { tionseinholung zunehmend unwichtiger werden } \\
\text { lässt. }\end{array}$ \\
\hline
\end{tabular}

\subsection{Aufhebung im Fokus}

Um Missverständnisse zu vermeiden, wollen wir das Verhältnis zwischen Aufhebung und Informationsupdating noch weiter spezifizieren: Einerseits kann, im Gegensatz zum rationalen Vertrauensgeber, der zur Aufhebung befähigte Vertrauensgeber auf intrusive Informationseinholung unmittelbar vor der Vertrauenshandlung, innerhalb der Reaktionszeit und zum Zeitpunkt der Vertrauenserwiderung verzichten. Denn er ist davon überzeugt, dass sich der Nehmer als vertrauenswürdig erweisen wird. Andererseits ist davon auszugehen, dass der Austausch von Informationen notwendig ist für die Bildung von Vertrauen und das Erlernen von Aufhebung - zumindest in den meisten Fällen. Akteure müssen sich gegenseitig als vertrauenswürdige Interaktionspartner erkennen und ein Gefühl der Vertrautheit entwickeln (Luhmann 2009: Kap. 3). ${ }^{25}$ Vertrautheit muss freilich mehr sein, als bloße Interaktionsgeschichte und kalte Information. Dies zeigt unsere Kritik am rationalistischen Lernmodell. Aus der Perspektive sozialpsychologischer Ansätze wird deshalb argumentiert, dass Vertrautheit zwischen Akteuren dann entstehen kann, wenn sie sich als Mitglieder ein und derselben moralischen Gemeinschaft wahrnehmen. Michael A. Hogg und Kollegen (Hogg 2000; Hogg et al. 2007) zeigen beispielsweise empirisch, dass Gruppenkategorisierung der Reduktion von Ungewissheit dient. Offenkundig fällt es Individuen leichter, Gruppenmitgliedern Vertrauen entgegenzubringen als Außenstehenden. ${ }^{26}$ Sozialisations- und Selbstkategorisierungsprozesse werden somit zur Basis von Vertrauen. Im Verlauf dieser Prozesse internalisieren Akteure die zentralen Werte und Normen einer Gruppe und können

25 Vincent Pouliot (2008: 278) theoretisiert Vertrauen als Beispiel für praktische Vernunft. In diesem Sinne können wir uns auch vorstellen, dass die Fähigkeit zur Aufhebung durch diese Handlungslogik theoretisiert werden könnte.

26 Siehe auch Yamagishi/Kiyonari (2000: 127). 
gruppenspezifische Verhaltenserwartungen herausbilden (Lewicki/Bunker 1995; Stolle 2002; Uslaner 2002; Mercer 2010; Williams 2001; Kramer 1999). ${ }^{27}$

Aus der Sozialpsychologie ist zudem bekannt, dass in-group-Vertrauen mit Wahrnehmungsverzerrungen und Vorurteilen einhergeht: Während konfrontatives Verhalten von Mitgliedern der eigenen Gruppe eher durch externe Zwänge erklärt und damit relativiert wird, werden vergleichbare Aktionen bei Fremden mit schlechten Charaktereigenschaften in Verbindung gebracht. Umgekehrt gilt, dass kooperatives Verhalten von Gruppenmitgliedern ihrem guten Charakter angerechnet wird, während freundliche Handlungen von Fremden grundsätzlich misstraut und auf mögliche böse Absichten untersucht werden. Die Gruppenzugehörigkeit beeinflusst also, wie objektiv identische Handlungen subjektiv unterschiedlich interpretiert werden. Diese Erkenntnis unterstreicht die existierende Kritik am rationalistischen Vertrauenskonzept: Offenbar justieren Vertrauensgeber ihre Vertrauenserwartung nicht auf objektive Weise im Lichte neuer Informationen, wie es die rationalistische Lerntheorie erwartet. Vielmehr werden neue Informationen nach Maßgabe bereits bestehender Vertrauenserwartungen unterschiedlich interpretiert (Taylor/Jaggi 1974; Jervis 1976; Hewstone et al. 1982; Pettigrew 1979).

Neben Gruppenkategorisierungen werden Emotionen als wesentliche Stellgrößen für Vertrautheit und Vertrauensbereitschaft identifiziert (Becker 1996; Jones 1996; Lahno 2001; 2002: Kap. 7) ${ }^{28}$ Nach Jack Barbalet (2009: 370, 375) braucht es einen emotionalen Trigger für Vertrauenshandlungen: »The basis of trust, then, is the feeling of confidence in another's future actions and also confidence concerning one's own judgment of another «. Vertrauen ist in dieser Sichtweise ein »emotional belief« (Mercer 2010), und damit sowohl kognitiv als auch affektiv begründet. Zwar können die beiden Dimensionen einen jeweils unterschiedlich hohen Anteil an Vertrauen haben, doch bedarf Vertrauen immer einer affektiven Komponente (Lewis/ Weigert 1985: 971). So konnten Michael Kosfeld et al. (2005: 674) in einer neurologischen Studie nachweisen, dass eine durch die Gabe des Hormons Oxytocin ausgelöste positive Emotion - ein »Gefühl der Wärme« gegenüber anderen Menschen - im spieltheoretischen Experiment eine signifikant höhere Vertrauensbereitschaft

27 Nach Eric Uslaner (2002: 18-19) existiert innerhalb von Identitätsgruppen sogar eine Norm zu vertrauensvollem Verhalten.

28 Nach Jonathan Mercer (2005: 95-96) bestehen zwischen Gruppenzugehörigkeit, Emotionen und Vertrauen enge Zusammenhänge: »I argue that identity produces emotion that creates trust $\ll$. 
zur Folge hatte (siehe auch Zak/Kugler 2011). ${ }^{29}$ Zusammenfassend zeigt sich, dass Vertrautheit durch eine positive Interaktionsgeschichte hergestellt wird, die zu wechselseitiger Identifikation und emotionaler Bindung als extra-rationale Relationseigenschaften führt. Aufhebung gründet wiederum auf Vertrautheit und lässt Schlüsse aus selektiven Erfahrungen auf die allgemeine Vertrauenswürdigkeit des Interaktionspartners zu.

Im Gegensatz zu Ansätzen, die die Bildung von Vertrautheit ins Zentrum stellen, stehen Studien, die individualpsychologische Dispositionen für ausschlaggebend halten (Rathbun 2011b; Uslaner 2002): Demnach mache es Sinn, Akteure nach Maßgabe ihrer Neigung zu generalisiertem Vertrauen zu typologisieren. Die einschlägige Literatur unterscheidet (Fischer/Wiswede 2009: 697) hier etwa zwischen »kooperativen«, »individualistischen« und »kompetitiven « Akteuren und zeigt, dass ihre Vertrauensbereitschaft unter ähnlichen Bedingungen signifikant variiert. Dementsprechend entsteht Vertrauen nicht allein auf der Basis von Vertrautheit, sondern erscheint im Sinne generalisierter Vertrauensbereitschaft als feststehende individualpsychologische Variable seitens des Vertrauensgebers.

\subsection{Die Relevanz der ontologischen Unterscheidung}

Unsere ausführliche ontologische Diskussion ist analytisch wichtig und empirisch folgenreich. Denn das Vertrauenskonzept würde jeden Mehrwert verlieren, wenn Vertrauenserwartungen nicht strikt auf akteursspezifische Eigenschaften bezogen werden. Andernfalls könnten wir Vertrauen als genuine und über Kontrolle hinausgehende Kooperationsressource überhaupt nicht sehen. Zudem ist es wichtig, dass Vertrauen in der Zuschreibung moralischer Dispositionen, Identifikation und emotionaler Nähe gründet. Auf diese Weise erreichen Vertrauensbeziehungen tatsächlich eine andere Qualität als egoistische Interessengemeinschaften. Gleichzeitig halten wir auch daran fest, Vertrauen als Aufhebungsentscheidung zu begreifen. Nur so lässt sich die extra-rationale Eigentümlichkeit unseres Untersuchungsgegenstandes angemessen erfassen und nur auf diese Weise lassen sich die irrationalen Momente, die bei Aufhebungsentscheidungen eine Rolle spielen, fokussieren. Wie gezeigt, weisen rationalismuskritische Ansätze auf sozialpsychologische Gruppenprozesse, Emotionen und Dispositionen als Basis von Vertrauen hin. Bleiben diese

29 In manchen Studien werden Emotionen im Kontext von Vertrauen auch als Erfahrungsspeicher konzeptualisiert. So begreift Maria Neumeier (2011: 294) Vertrauen als Form »verdichteten Wissens« (bzw. als »somatischen Marker «), welches sich aus vergangenen Erfahrungen bildet und in ähnlichen Situationen abgerufen und auf solche übertragen wird. Mit zunehmender Erfahrung entsteht eine stabile Verhaltensdisposition, die es ermöglicht, in bestimmten Situationen »kognitiv unaufwändig zu vertrauen«. Dieser Prozess läuft dabei zu einem erheblichen Teil im Unbewussten ab: »Prozesse ohne Beteiligung des Bewusstseins sind besonders schnell und werden subjektiv als besonders sicher empfunden«. Zudem kann eine emotionale Bindung als Basis für Vertrauen erklären, warum Vertrauensenttäuschung als Treuebruch oder Verrat empfunden werden, während in kalkülbasierten Interaktionen, in denen Vertrauen keine Rolle spielt, unkooperatives Verhalten als reine Enttäuschung wahrgenommen wird (Michel 2012: 13). 
Faktoren in theoretischen Modellen und ihren empirischen Überprüfungen unberücksichtigt, laufen sie Gefahr, an Erklärungs- und Aussagekraft zu verlieren. Beispielsweise kann eine rein rationalistische Lerntheorie Vertrauensbildungsprozesse kaum angemessen erfassen und übersieht wichtige Bedingungen für ihren Erfolg.

\subsection{Vertrauen als Komplementärmechanismus}

Unsere starke Kontrastierung von kontroll- und vertrauensbasierter Kooperationserwartung hat vor allem heuristischen Wert. Deshalb plädieren wir in der empirischen Forschung für einen pragmatischen Umgang mit der Frage, aus welchen Gründen Akteure zusammenarbeiten. Denn in unserem Verständnis schließen sich Kontrolle und Vertrauen im strengen Sinne nicht wechselseitig aus. Hier folgen wir Anthony Giddens (1985): Soziale Akteure reproduzieren in ihrer Praxis soziale Strukturen was wir wissen können -, gleichzeitig sind sie aber immer auch für Überraschungen gut - womit wir rechnen sollten. Ob diese Überraschungen aber mit Respekt vor den legitimen Interessen anderer einhergehen, darauf können wir nur vertrauen. Denn Akteure haben bei aller Strukturiertheit ihrer Handlungen immer Entscheidungsspielräume, in denen sie sich als moralische Akteure bewähren könn(t)en. Hieraus folgt für die Kooperationsforschung, dass Kontrolle und Vertrauen bei allen begrifflichen Unterscheidungen in komplementärer Weise die Bildung positiver Erwartungen anleiten (siehe Abbildung 3). Niemand wird bestreiten, dass die antizipierte Wirkung von Kontrollmechanismen - sei es in Form struktureller Einschränkungen oder sei es in Form kontinuierlicher Informationseinholung - in der internationalen Politik für die Herausbildung positiver Erwartungen wichtig ist. Gleichzeitig wollen wir aber auch die Möglichkeit nicht außer Acht lassen, dass Akteure auf Kontrollmechanismen verzichten, da sie einander vertrauen. Wenn aber Vertrauen in einem Komplementärverhältnis zu Kontrollmechanismen steht, dann ist nicht mehr von Interesse, ob Akteure vertrauen oder kontrollieren, sondern wie viel von der objektiv existierenden Ungewissheit aufgehoben werden kann, und damit einhergehend wie viel strukturelle Kontrolle oder Informationseinholung notwendig ist, damit Akteure robuste Kooperationserwartungen bilden können: Welche Kontrollmechanismen werden institutionalisiert, wie viel Informationen werden eingeholt und verarbeitet, und wie stark wird dem Partner vertraut? Die Frage nach dem Mischungsverhältnis von Vertrauen und Kontrolle tritt in den Vordergrund. In diesem Sinne verringert ein Mehr an Vertrauen die Notwendigkeit von Kontrollmechanismen, um positive Erwartungen herausbilden zu können. Wir machen uns somit die umgangssprachliche Intuition zu Nutze, dass Vertrauen - auch zwischen Staaten - wachsen kann. Wir haben es deshalb nicht mit einer Dichotomie wie beim strategischen und kommunikativen Handeln $\mathrm{zu}$ tun, sondern mit einem realweltlichen Phänomen, das mehr oder weniger stark ausgeprägt sein kann. In diesem Sinne umgehen wir auch die meta-theoretische Zwickmühle der ZIB-Debatte (Deitelhoff/Müller 2005; Herborth 2007; Risse 2007). 


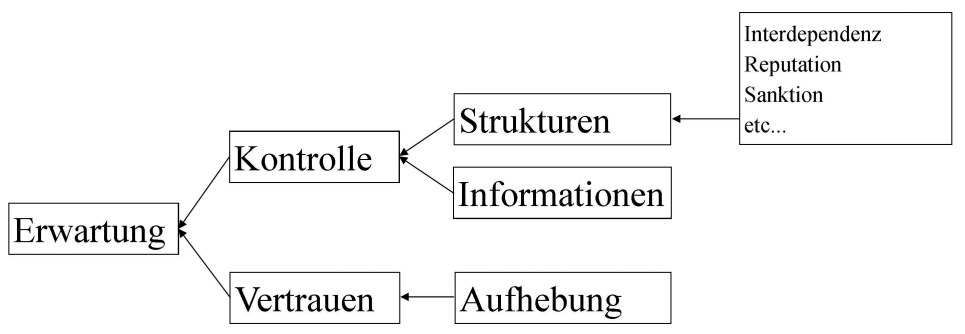

\section{Vertrauen in den $I B$}

\subsection{Vertrauen als Kalkül in den $I B$}

Rationalistische Ansätze zur internationalen Politik schätzen die Bedeutung von Vertrauen höchst unterschiedlich ein. So geht der offensive Neorealismus von aggressiven Machtmaximierern aus, auf deren Kooperationszusagen kein Verlass ist. Deshalb sollten Staaten ihrem Außenverhalten Worst-Case-Szenarien zugrunde legen. Entsprechend formuliert Mearsheimer (2001: 32): »There is little room for trust among states. For sure, the level of fear varies across time and space, but it cannot be reduced to a trivial level«. Wesentlich optimistischer sind defensive Neorealisten. Sie modellieren Staaten nicht als Macht- sondern als Sicherheitsmaximierer, die einander dann vertrauen können, wenn sie sich in einem entspannten Umfeld ohne vitale Konflikte bewegen (Glaser 1994: 71).

Der bayesianische Realismus von Kydd (2005: Kap. 1, 2) greift zentrale Annahmen der beiden neorealistischen Spielarten auf und verknüpft sie mit Intuitionen des neoklassischen Realismus. Demnach können Staaten entweder ausbeuterische Expansionisten oder kooperationsbereite Sicherheitsmaximierer sein. Letztere müssen sich allerdings als solche erkennen, bevor sie vertrauensvoll kooperieren können. Dies wird durch relativ einfach modellierte Signalspiele erreicht, die sich stark an Charles E. Osgoods psychologisch fundiertem Graduated Reciprocation in Tension-Reduction Modell (GRIT) orientieren (Lindskold 1978; Osgood 1962). Dementsprechend gleicht Kydds Vertrauenskonzeption am ehesten einer psychologisierten Rationalismusvariante. Staaten können ihr Interesse an kooperativen Beziehungen und ihre Vertrauenswürdigkeit kommunizieren, indem sie zeigen, dass sie bereit sind, riskante Vorleistungen zu erbringen. Solche Vorleistungen werden als kostspielige Signale modelliert, die meist die Form unilateraler kooperativer und riskanter Gesten annehmen. Das im Rahmen einer ersten Signalrunde aufgebaute Vertrauen bildet die Grundlage für weitergehende Zusammenarbeit. Dabei ist zu beachten, dass nur solche Signale glaubwürdig sind, zu deren Sendung ausschließ- 
lich vertrauenswürdige Staaten bereit sind. Sie sollen zeigen, dass für den Senderstaat die potentiellen Gewinne von Kooperation das Risiko der Signalrunde kompensieren und deshalb mit seiner zukünftigen Zuverlässigkeit gerechnet werden kann. Signale, die diesen Anforderungen nicht gerecht werden, sind als cheap talk bedeutungslos. ${ }^{30}$ In drei Fallstudien zum Beginn des Kalten Krieges, zur Gründung der NATO und zum Ende des Kalten Krieges arbeitet Kydd die Analysekraft seiner formalen Theorie heraus (Kydd 2005: Kap. 5-8). ${ }^{31} \mathrm{Zu}$ erwähnen ist in diesem Zusammenhang, dass Signalspiele bei der Bildung aufhebungsbasierten Vertrauens eine wichtige Rolle spielen. Ob sie allerdings lediglich Erwartungssicherheit durch glaubwürdige Informationen fördern und damit die Grundlage für langfristige Annäherungsprozesse bilden, oder ob sie in einem genuinen Sinne aufhebungsbasiertes Vertrauen erzeugen können, ist strittig (siehe unten). Dementsprechend bleibt die Frage ungeklärt, ob die Signale in Kydds Fallbeispielen Vertrauen erzeugen konnten.

Auch in der neoliberalen Kooperationstheorie spielt rationalistisches Vertrauen eine wichtige Rolle zur Ermöglichung von Zusammenarbeit unter den Bedingungen von Anarchie und Interdependenz (Keohane 1986; 1993; Ostrom 2002). Brian C. Rathbun argumentiert sogar, dass Vertrauen das Fundament der rationalistischen Kooperationstheorie darstellt. Meistens wird Vertrauen dabei lediglich implizit konzeptualisiert:

»This is the kind of trust utilized, often only implicitly, in rationalist work in international relations. It provided the foundation of what was known as >neoliberal institutionalism< [...] (Rathbun 2009: 350).

Es kann tatsächlich argumentiert werden, dass rationalistisches Vertrauen in allen Ansätzen mitgedacht werden muss, in denen auf der Basis von Informationen Unsicherheit minimiert und Kooperation ermöglicht wird. Im Gegensatz zu den psychologisierten Theorien von Osgood und Kydd werden diese Informationen im Neoinstitutionalismus jedoch weniger durch kostspielige Signale als durch die transparenzschaffende Wirkung internationaler Institutionen erzeugt. Sie organisieren einen kontinuierlichen Informationsfluss und sorgen für deren zuverlässige Auswertung. So dienen der Open Skies Treaty oder das Wiener Dokument der Überprüfung von Regeleinhaltung und der Identifikation von Regelbrüchen bei Rüstungskontrollmaßnahmen. Selbiges gilt für zahlreiche internationale Organisationen wie

30 Harald Müller argumentiert gegen die Effektivität von costly signalling zur Herstellung von rationalistischem Vertrauen, da der bloße Empfang kostspieliger Signale nicht die Vermutung unbekannter privater Informationen auf Vertrauensnehmerseite schwächen kann (Müller 2004: 398). Die Theorie des costly signalling sei somit auf Annahmen angewiesen (etwa ein geteiltes Verständnis von Sicherheit und Kosten), die nicht selbstverständlich seien. Aaron M. Hoffman (2007: 302-205) merkt zudem an, dass Staaten, die sich ernsthaft misstrauen, nicht bereit sein werden, das Risiko überzeugender kostspieliger Signale einzugehen.

31 Weitere oft zitierte empirische Beispiele für die vertrauensbildende Wirkung von kostspieligen Signalen sind die Rede Anwar Sadats vor der israelischen Knesset 1977, der Besuch des indischen Premiers Atal Bihari Vajpayee im pakistanischen Lahore sowie die einseitigen Abrüstungsbemühungen im Rahmen des Österreichischen Staatsvertrags (Larson 1997a; 1997b; Wheeler 2010). 
der Internationalen Atomenergiebehörde, die in ihren praktischen Funktionen als Informationsdienstleister theoretisiert werden und somit der Herstellung von rationalistischem Vertrauen dienen. ${ }^{32}$ Dass es sich hierbei im Kern um kontroll- und informationsbasierte und weniger um eigenschaftsbasierte Kooperationserwartungen handelt, bleibt unbenommen.

Informationsbasiertes Vertrauen gilt darüber hinaus als zentrale Bedingungen für die erfolgreiche Zusammenarbeit im iterierten Gefangenendilemma. Damit die von Robert Axelrod (1984: 174, 182) als Tit-for-Tat bekannte Interaktionsstrategie kooperationsermöglichend wirkt, müssen Staaten zum einen über das vergangene Kooperationsverhalten Bescheid wissen, um im nächsten Spielzug entsprechend reagieren zu können. Zum anderen sollten die Akteure hinreichend über die Interdependenz und den damit verbundenen langen Schatten der Zukunft informiert sein. Schließlich müssen sie ein Mindestmaß an Kooperationsbereitschaft der anderen Seite erwarten können, damit sie reziproke Austauschbeziehungen überhaupt eingehen und kooperative Strategien wählen können. ${ }^{33}$ Folglich legen die Akteure ihren Kooperationsentscheidungen subjektive Wahrscheinlichkeitseinschätzungen zur Robustheit der Dilemmastruktur und der Rationalität ihrer potentiellen Partner zu Grunde, die als rationalistisches Vertrauen interpretiert werden können (Kydd 2005: 7-11). ${ }^{34}$

Insgesamt lässt sich festhalten, dass rationalistisches Vertrauen in der einschlägigen Literatur oftmals implizit mitgedacht, aber kaum explizit theoretisiert und analysiert wird. Eigentlich stellen in dieser Hinsicht nur die Arbeiten von Kydd eine Ausnahme dar. Und selbst Kydd legt seinen Überlegungen keine strengen Rationalitätsannahmen zu Grunde, sondern wählt eine weichere, psychologisierte Rationalismusvariante. Ansonsten gilt, dass Vertrauen in der rationalistischen Kooperationstheorie informationsbasierte und kurzfristige Erwartungssicherheit ermöglicht. Auf diesem Weg findet zwar eine indirekte Beschäftigung mit dem Vertrauensphänomen statt, allerdings bringt das Konzept nichts wirklich Neues für die Analyse internationaler Politik. $\mathrm{Zu}$ dieser Schlussfolgerung kommt auch Mercer: »If trust depends on external evidence, transparency, iteration, or incentives, then trust adds nothing to the explanation« (Mercer 2005: 95). ${ }^{35}$

32 Vertrauensbildende Maßnahmen erlauben nicht nur die Bildung von rationalistischem Vertrauen durch Informationseinholung. Sie wirken auch vertrauensbildend, indem sie als costly signal verstanden werden, da die Preisgabe militärisch sensibler Informationen durchaus mit Risiken verbunden ist. Ob vertrauensbildende Maßnahmen auch zur Erzeugung aufhebungsbasierten Vertrauens dienen können, ist eine bislang wenig erforschte Fragestellung.

33 Dies gilt vor allem, wenn Defektion des Interaktionspartners einen überproportional großen Schaden mit sich bringt.

34 Kydds (2005) Studien sind eine der wenigen empirischen Beiträge, die das Konzept des rationalistischen Vertrauens explizit empirisch verwenden, siehe aber auch Alex Gillespie (2012).

35 Siehe auch Rathbuns (2009) Kritik am rationalistischen Vertrauenskonzept. 


\subsection{Vertrauen als Aufhebung von Ungewissheit in den IB}

Vertrauen im Sinne der Aufhebung von Ungewissheit scheint in der internationalen Politik auf den ersten Blick ein seltenes Gut zu sein. Gleichwohl zeigt ein zweiter Blick, dass es in unterschiedlichen Forschungszusammenhängen entweder implizit vorausgesetzt oder explizit thematisiert wird. Insgesamt identifizieren wir mehrere Bereiche in den IB, die sich auf die eine oder andere Art und Weise mit aufhebungsbasiertem Vertrauen auseinandersetzen: die Forschung zu internationaler Gemeinschaftsbildung, die psychologisch fundierte Außenpolitikforschung, die Versöhnungsforschung sowie jüngere Forschungsansätze zu Respekt und Freundschaft.

Booth und Wheeler (2008) gehören zu jenen Autoren, die sich bislang am intensivsten mit aufhebungsbasiertem Vertrauen in der internationalen Politik auseinandergesetzt haben. ${ }^{36}$ In ihrem konzeptionell starken Buch beschreiben sie Ungewissheit unter anarchischen Bedingungen als Konstante, der durch gemeinsame Informationspolitik und robuste Kontrollmechanismen nicht beizukommen sei. Vielmehr lasse sich die Logik des Sicherheitsdilemmas dauerhaft nur durch Vertrauen überwinden. Hier nähern sich die beiden Autoren unserem Idealtyp von Vertrauen an und fokussieren sein extra-rationales Element, indem sie Empathie, emotionale Bindung und integeres Verhalten als zentrale Eigenschaften von Vertrauensbeziehungen herausstellen. Die Bildung eines Vertrauens, das geeignet ist, das Sicherheitsdilemma $\mathrm{zu}$ überwinden, beginne mit einem »leap in the dark« (Booth/ Wheeler 2008: 234; Wheeler 2010). Dieser »Sprung ins Dunkle« wiederum wird von den beiden Autoren als kostspieliges Signal im Sinne Kydds begriffen. Er beschreibe einen subjektiv riskanten »frame-breaking conciliatory move« und helfe, den Interaktionspartner von der eigenen kooperativen Absicht zu überzeugen (Wheeler 2010: 326, Fn. 19). Ohne eine solche riskante Vorleistung lassen sich Dilemmasituationen nach Überzeugung von Booth und Wheeler nicht durchbrechen. Als weitere Bedingungen für erfolgreiche Vertrauensbildung nennen die beiden Autoren eine wechselseitige Sensibilität für die fatalen Dynamiken des Sicherheitsdilemmas und die Entwicklung langfristiger gemeinsamer Interessen und geteilter Werte auf transnationaler Ebene (Booth/Wheeler 2008: 234).

36 Neben Booth und Wheeler hat auch Hoffman $(2002 ; 2006 ; 2007)$ einen wichtigen Beitrag zur Vertrauensforschung in den IB geleistet, der vor allem im Hinblick auf konzeptionelle Überlegungen und auf die Operationalisierung von Vertrauensbeziehungen bedeutsam ist. Seine theoretischen Überlegungen zu Vertrauensbildungsprozessen, die er auch in Fallstudien zum institutionellen Design der Europäischen Gemeinschaft und zur Wasserverteilung zwischen Israel, Jordanien und den palästinensischen Gebieten empirisch überprüft, wirken allerdings konfus. Entscheidend für die Überwindung von Misstrauen und dem Aufbau von Vertrauen sind nach Hoffmann internationale Institutionen. Sie hätten das Potential, Unsicherheiten soweit abzumildern, dass Raum für Vertrauensverhältnisse entsteht. Allerdings bleibt das Verhältnis von Institutionen und Vertrauen ungeklärt. Manche Passagen, vor allem solche in seinem Buch, lesen sich so, als ob Institutionen Kontrollmechanismen etablieren und diese Kontrollmechanismen eine notwendige Voraussetzung für Vertrauen sind. Vertrauen droht damit über die Hintertür von Institutionen wieder seine ontologische Eigenständigkeit zu verlieren, da funktionierende Beziehungen im Letzten auf Kontrolle beruhen. 
Vor dem Hintergrund ihres Vertrauenskonzepts kritisieren Booth und Wheeler (2008: 155) Kydds rationalistische Beschreibung von Vertrauensbildungsprozessen, die das Ende des Kalten Krieges einleiteten: Kydd sehe nur kostspielige Signale, wo in Wirklichkeit schon Vertrauen geherrscht habe, da er die bereits erfolgte emotionale Annäherung und Bindung der Staatschefs in seinem rationalistischen Modell nicht greifen könne. Problematisch an dieser Kritik bleibt allerdings, dass Booth und Wheeler keine strukturierten und belastbaren Indikatoren für Vertrauen angeben. Vielmehr verlassen sich die Autoren auf ihre eigene Interpretation von Handlungen und Aussagen der Akteure. Ohne valide und reliable Indikatoren lassen sich vertrauensbildende kostspielige Signale jedoch nicht von genuinen Vertrauenshandlungen unterscheiden. Beobachtet man das bloße Handeln der Akteure scheinen beide Interpretationen zulässig.

Trotz dieser methodologischen Schwäche können die Fallstudien zu den OsloVerhandlungen (Booth/Wheeler 2008: 245-251), zur Annäherung zwischen Indien und Pakistan (Wheeler 2010) und zur Überwindung des nuklearen Rüstungswettlaufs zwischen Argentinien und Brasilien durch die Institutionalisierung eines effektiven bilateralen Verifikationsregimes (Wheeler 2009) als Plausibilitätsproben für die Notwendigkeit von Vertrauen für die Überwindung des Sicherheitsdilemmas dienen. Sie legen darüber hinaus nahe, dass der sprichwörtliche Sprung ins Dunkle selbst unter widrigen Bedingungen Vertrauensbildungsprozesse in Gang setzen kann. Wobei die drei Fallstudien - und hier vor allem die Untersuchung zum Oslo-Prozess - auch deutlich machen, dass dieser Sprung scheitern kann. Schließlich bleibt strittig, ob das Vertrauenswagnis in den drei Fällen tatsächlich zu robustem Vertrauen zwischen den Akteuren geführt hat. So wendet Torsten Michel (2012: 9) ein, dass der Aufbau intrusiver Kontrollmechanismen zur Beendigung der argentinisch-brasilianischen Rüstungsrivalität eher für weniger Misstrauen als für mehr Vertrauen spreche. Ähnliches ließe sich mit Blick auf den Oslo-Prozess und die indisch-pakistanische Annäherung sagen. Umgekehrt kann aber mit Booth und Wheeler argumentiert werden, dass die Akzeptanz von Verifikationsrechten die Preisgabe sensibler Informationen impliziere und in diesem Sinne ein erhebliches sicherheitspolitisches Wagnis darstelle, welches vertrauensvolle Partner voraussetze (siehe auch die Position der Sowjetunion zu Verifikation als Form legitimierter Spionage (Kokeyev/Androsov 1990)).

Weitere Arbeiten, die zwischenstaatliches Vertrauen und Gemeinschaftsbildung in Zusammenhang bringen, knüpfen an sozialpsychologische Forschungen zu ingroup-Dynamiken an. So kann nach Alexander Wendt langanhaltende Kooperation zur Herausbildung kollektiver Identitäten führen, wie das Beispiel der westeuropäischen Integration zeige. Solche Identitäten gingen mit einem starken Wir-Gefühl einher. Auf dessen Grundlage würden Akteure immer wieder füreinander einstehen, ohne dass dies für sie selbst mit einem unmittelbaren Vorteil verbunden wäre. Allerdings seien Staaten hierzu nur bereit, wenn sie nicht fürchten müssten, von anderen Gruppenmitgliedern in Schwächephasen übervorteilt zu werden (Wendt 1999: 357-363). In diesem Sinne setzt kollektive Identität Vertrauensbildung voraus und geht mit Vertrauen einher. Wendt zufolge zeichnen sich erfolgreiche Inte- 
gration und Gemeinschaftsbildung also dadurch aus, dass die Gruppenmitglieder voneinander Solidarität erwarten, ohne dass sie zuverlässig von außen erzwungen oder motiviert werden könnte:

»External constraints may play a role in initiating the building of trust, but collective identity implies giving over to the Other at least some responsibility for the care of the Self, and that generally requires [...] a belief that the Other will constrain itself in the demands it makes on the self « (1999: 359, Hervorh. dort).

Gruppenidentität und Vertrauen werden auch in den Untersuchungen zu internationalen Sicherheitsgemeinschaften verknüpft. ${ }^{37}$ Für Sicherheitsgemeinschaften sind generalisierte Erwartungen friedlicher Konfliktbeigelegung unter den Mitgliedern charakteristisch. Diese Erwartung wird von einer starken, kollektiven Identität getragen (Adler/Barnett 1998a: 45-48). Vertrauen wiederum erscheint als Überzeugung, dass sich andere Staaten in einer komplexen Welt aus innerem Antrieb bei der Bearbeitung von Interessengegensätzen an gemeinsamen Werten und Normen orientieren werden. Es bildet sich ähnlich wie bei Wendt in langfristigen Lernprozessen heraus, in deren Verlauf sich eine situationale, an strategischen Kalkülen ausgerichtete Orientierung der Akteure in eine dispositionale Orientierung verändert, die auf moralische Integrität und eine geteilte Weltdeutung setzt. Aus dieser Perspektive wird die rationalistische Konzeptualisierung von Vertrauen also nicht pauschal verworfen, sondern perspektiviert. ${ }^{38}$ Aufhebungsbasiertes Vertrauen steht am Ende eines weitgehend durch kalkulative Logik geprägten Annäherungsprozesses. Demnach kann anhaltende Kooperation zwischen egoistischen Nutzenmaximieren Konsequenzen für eben diese Nutzenmaximierer haben, indem sie zur Ausbildung kollektiver Identitäten führt. Aus dieser Sicht spielen internationale Institutionen zwar bei der Vertrauensbildung durchaus eine wichtige Rolle, indem sie Erwartungen durch Regelsetzung und Regelüberwachung in interdependenten Beziehungen stabilisieren. Ihre Bedeutung sinkt aber in dem Maße, in dem sich eine robuste Sicherheitsgemeinschaft etabliert hat: »States no longer rely on concrete international organizations to maintain trust but do so through knowledge and beliefs about the other (Adler/Barnett 1998a: 46). Hinsichtlich seines empirischen Ertrags bleibt das Programm zu Sicherheitsgemeinschaften leider weit hinter seinen fundierten theoretischen Erwartungen zurück. Wenn überhaupt, dann wird Vertrauen in den Fallanalysen als peripherer Faktor erwähnt, dessen genaue kausale Rolle nicht näher untersucht wird. Dies gilt auch für spätere Fallanalysen (Adler/Greve 2009; Flemes 2003; Jones 2008; Kacowicz 2000).

Da sich bis heute Sicherheitsgemeinschaften meist zwischen Demokratien entwickeln, vermutet die konstruktivistische Forschung zum demokratischen Frieden einen Nexus zwischen demokratischer Gemeinschaft und zwischenstaatlichem Vertrauen (Müller/Wolff 2006; Risse-Kappen 1995). Allerdings erscheint Vertrauen hier weniger als Ergebnis langfristiger Lern- und Sozialisationsprozesse, sondern

37 Vgl. Adler (1997); Adler/Barnett (1998a; 1998b); Barnett/Adler (1998); Bengtsson (2000); Kacowicz et al. (2000); Kupchan (2010); Väyrynen (2000).

38 Siehe auch das Modell zu Vertrauensbildung von Roy J. Lewicki und Barbara B. Bunker (1995). 
entsteht unmittelbarer auf der Grundlage geteilter Werte und Normen. Demnach erkennen sich Demokratien aufgrund ihrer ähnlichen innenpolitischen Strukturen als Demokratien und wissen, dass gewählte Regierungen ihre Gesellschaften niemals von der Notwendigkeit eines Krieges gegen eine andere Demokratie würden überzeugen können. Gewalt sei allenfalls zur Verteidigung oder Verbreitung demokratischer Werte zulässig und deshalb seien Kriege zwischen Demokratien ausgeschlossen.

In der psychologisch fundierten Außenpolitikanalyse lassen sich weitere Anknüpfungspunkte für ein aufhebungsbasiertes Vertrauenskonzept finden. So setzt Rathbun (2011a; 2011b; 2011c) die generalisierte Vertrauensdisposition von außenpolitisch Verantwortlichen mit ihren Weltbildern und Überzeugungen in Beziehung. Bemerkenswert ist in diesem Zusammenhang, dass sich die Ausprägung von Vertrauensdispositionen relativ präzise über die Nähe zu realistischen oder konstruktivistischen Theoremen bestimmen lässt. So zeigt die Analyse der außenpolitischen Einstellungen von Republikanern und Demokraten im amerikanischen Kongress nach 1945, dass bei Republikanern Misstrauen, Unilateralismus und Realismus zusammengingen, während Demokraten ihre eher kooperative und vertrauensvolle Orientierung mit einer intuitiven Neigung zu konstruktivistischen Argumenten verbanden. Diese Unterschiede in der generellen Vertrauensneigung spiegeln sich nach Rathbun (2011b: 329) in Präferenzen über das Design internationaler Institutionen wider. Während Akteure mit einer ausgeprägten generalisierten Vertrauensdisposition sich eher für prinzipienorientierte, multilaterale Designs einsetzten, die der Logik diffuser Reziprozitätsspiele folgten, wollten Akteure mit geringer Vertrauensdisposition nur Institutionen mit schwachen Verbindlichkeiten akzeptieren, die ihnen starke Vetomöglichkeiten einräumten und auf spezifischer Reziprozität basierten. ${ }^{39}$ Entsprechend waren Demokraten unter identischen, internationalen Rahmenbedingungen auch eher bereit für die NATO in bündnispolitische Vorleistung zu treten als die Republikaner, die fürchteten, dass die Europäer ein amerikanisches Engagement opportunistisch ausnutzen und ihre eigenen Verteidigungsanstrengungen reduzieren würden. Auf Basis weiterer Fallbeispiele zur Gründung des Völkerbunds und der UNO argumentiert Rathbun, dass internationale Institutionen nicht Ausdruck fehlenden Vertrauens seien, wie anderswo behauptet (Koremenos et al. 2001: 766). Vielmehr setzen sie immer wieder ein erhebliches Maß an Vertrauen

39 Nach Robert O. Keohane (1986: 21) verwandelt sich spezifische Reziprozität im Zuge erfolgreicher Zusammenarbeit in diffuse Reziprozität. Staaten erwarten dann nicht mehr unmittelbare Gegenleistungen, sondern halten sich an Vereinbarungen und gemeinsame Regeln, weil sie davon ausgehen, dass auch andere Staaten in vergleichbaren Situationen eben diese Vereinbarungen und Regeln respektieren werden, und deshalb Kosten wie Nutzen der regelorientierten Zusammenarbeit gleichmäßig verteilt sind. In diesem Sinne stehen Vertrauen und diffuse Reziprozität in einem unmittelbaren Zusammenhang, wobei wir mit Rathbun übereinstimmen und Vertrauen als Grundlage für diffuse Reziprozitätserwartungen betrachten. 
voraus (vgl. auch Jönsson 1993) für die Rolle von kommunikativ hergestelltem Vertrauen bei der Errichtung internationaler Regime. ${ }^{40}$

Weitere Anknüpfungspunkte für eine anspruchsvolle Vertrauensforschung finden sich in Arbeiten zu images in der Außenpolitik. ${ }^{41}$ Beispielweise kann Vertrauen als konstitutives Element des ally image verstanden werden. Empirische Forschungen bestätigen den Zusammenhang zwischen Aktivierung des ally image und Vertrauensbildung. So ergeben Rezarta Bilalis (2010: 287) Feldexperimente starke Korrelationen zwischen der Aktivierung des ally images und Vertrauen, Respekt, und Bewunderung. Wichtig ist dabei, dass auch in der image-Forschung unsere konzeptionelle Unterscheidung besteht: Kooperative Erwartung können auf dem Wissen über Egoismus oder Integrität des Anderen basieren (Herrmann et al. 1997: 408-411).

Darüber hinaus wird aufhebungsbasiertes Vertrauen in der Versöhnungsforschung thematisiert. Die Rolle von Vertrauen wird dabei meist mit Hilfe von Feldexperimenten in Nachkriegsgesellschaften untersucht. So können Arie Nadler und Ido Liviatan (2006: 467-469) zeigen, dass im Rahmen israelisch-palästinensischer Friedensprojekte Äußerungen gegenseitiger Empathie und Schuldeingeständnisse nur dann zu erhöhter Versöhnungsbereitschaft führen, wenn sich die verfeindeten Gruppen ein Mindestmaß an Vertrauen entgegenbringen. Die Analyse nordirländischer Versöhnungsprojekte von Miles Hewstone et al. (2008) ergibt, dass unkooperatives Verhalten innerhalb der eigenen in-group zu drastischeren Vertrauensbrüchen führt, als dies für in-group-out-group-Interaktionen der Fall ist. In einer weiteren Studie belegen sie zudem den durch die inter-group-contact-Hypothese postulierten Zusammenhang zwischen regelmäßiger Interaktion und Vertrauensbildung (für eine Zusammenfassung dieser Forschungen siehe Hewstone et al. (2008)). Hieran anlehnend betonen John F. Dovidio et al. (2008: 233-236) den Zusammenhang zwischen regelmäßiger kooperativer Interaktion, den dadurch aktivierten Prozess der Rekategorisierung ehemals antagonistischer Gruppenidentitäten zu einer neuen und gruppenübergreifenden »Superidentität« und der Bildung von Vertrauen. ${ }^{42}$ Eher abstrahierend theoretisiert Reuben M. Baron (2008: 287) die kausale Verortung von Vertrauen in Versöhnungsprozessen, und kommt zu dem Ergebnis, dass ein Mindestmaß an Vertrauen als Bedingung für Versöhnung, gesteigertes Vertrauen als Folge von Versöhnung begriffen werden kann. Einen anderen Ansatz folgen Trudy Govier und Wilhelm Verwoerd (2002: 185-186). Für sie ist eine kausalanalytische Trennung von Vertrauensbildung und Versöhnung unzulässig, vielmehr ist Versöhnung nichts anderes als Vertrauensbildung. Die Autoren zeigen dabei, dass die in der Literatur gängigen Konzeptionen und Zieldefinitionen von Ver-

40 Einzelne Studien haben auch versucht, generalisiertes Vertrauen und damit verbundene Perzeptionen von Fremdstaaten auf Bevölkerungsebene zu erheben (Brewer et al. 2004).

41 Alexander et al. (1999); Brewer/Alexander (2002); Herrmann (1998); Herrmann (2003); Herrmann et al. (1997).

42 Siehe Nadler und Saguy (2004); Raudsepp und Wagner (2012); Rubini und Palmonari (2012). 
söhnung (Friendship, Cooperation, Peaceful Coexistence) allesamt, entweder implizit oder explizit, Vertrauensbildung als notwendigen Bestandteil begreifen.

Schließlich wollen wir noch auf zwei jüngere Forschungsprogramme hinweisen, in denen aufhebungsbasierte Varianten von Vertrauen eine Rolle spielen. In der Forschung zu internationalen Freundschaften ist Vertrauen ein zentrales Konzept, dem das Potential zugesprochen wird, positive Kooperationsspiralen zu aktivieren und aufrecht $\mathrm{zu}$ halten. Allerdings zeigen die vorliegenden Veröffentlichungen auch, dass noch Uneinigkeit über die Ontologie zwischenstaatlicher Freundschaftsbeziehungen herrscht und damit wiederum unklar ist, ob zwischenstaatliches Vertrauen notwendige Bedingung, integraler Bestandteil oder das Resultat von Freundschaft zwischen Staaten darstellt (für einen Überblick zu Freundschaft in den internationalen Beziehungen siehe Andrea Oelsner und Antoine Vion (2011)). Gegenteiliges gilt für die Forschungen zu Respekt als Determinante stabiler zwischenstaatlicher Kooperation (Wolf 2008; 2011: 122-123). Kausaltheoretisch wird hier Vertrauen eine explizite intervenierende Rolle zugeschrieben: Respekt stellt eine notwendige Bedingung für die Entstehung von Vertrauen dar, wobei Vertrauen wiederum robuste Kooperation im anarchischen Umfeld ermöglicht. Wenn Respekt sicherlich als notwendige, jedoch nicht als hinreichende Bedingung für Vertrauen verstanden werden kann, so muss Reinhard Wolfs Plädoyer für eine tiefergehende Auseinandersetzung mit Respekt als indirektes Plädoyer für die Beschäftigung mit weiteren Entstehungsbedingungen zwischenstaatlichen Vertrauens verstanden werden.

Zusammenfassend lässt sich festhalten, dass Vertrauen, verstanden als Aufhebung von Ungewissheit, in unterschiedlichen Bereichen der IB thematisiert wird. Die Hauptliteratur lässt sich dabei auf einen begrenzten Autorenstamm eingrenzen, wobei sich in hochrangigen Journals bislang nur wenige Beiträge zu aufhebungsbasiertem Vertrauen finden lassen. Eine tiefergehende ontologische Auseinandersetzung mit der Frage, was zwischenstaatliches Vertrauen ist, was es sein kann und zwischen wem es sich herausbildet, ist noch Mangelware. Empirische Analysen liegen kaum vor. Dies überrascht nicht zuletzt deshalb, als Vertrauen in Theorie und Praxis immer wieder eine herausragende Bedeutung für ein friedliches soziales Miteinander zugesprochen wird. Wir führen daher das Fehlen fundierter Vertrauensforschung weniger auf eine allgemeine Vertrauensskepsis innerhalb der wissenschaftlichen Community zurück, sondern sehen vielmehr die anspruchsvolle Konzeptualisierung und den erschwerten empirischen Zugang zu Vertrauen als Hauptgrund hierfür. Was nach wie vor fehlt, sind überzeugende Indikatoren für Vertrauen zwischen Staaten. ${ }^{43}$

43 Für Experimentalstudien gilt diese Feststellung freilich nicht. Hier werden die etablierten Fragebogen-Items aus der Soziologie und Sozialpsychologie für die Erfassung von Vertrauenserwartungen verwendet. 


\section{Der empirische Zugang zu Vertrauen als Aufhebung}

Generell spricht einiges dafür, dass rationalistische Vertrauensontologien mit unzulässigen Annahmen arbeiten und weitverbreiteten Intuitionen dessen, was Vertrauen ausmacht, nicht gerecht werden. Ob es allerdings Vertrauen, verstanden als Aufhebung von Ungewissheit, tatsächlich in der generell als vertrauensfeindlich eingeschätzten internationalen Politik gibt, ist damit noch lange nicht gesagt. Eine plausible theoretische Annahme ersetzt keinen empirischen Nachweis. Ein solcher Nachweis von Vertrauen ist nicht zuletzt deswegen so schwierig, weil sich Vertrauen als Einstellung nicht unmittelbar beobachten lässt. Wendt (1999: 360) spricht in diesem Zusammenhang auch von unserer »inability to read others' minds «. Wenn überhaupt, dann sind solche nicht beobachtbaren Handlungsdispositionen über den Umweg von Verhaltensauffälligkeiten erfassbar (vgl. Deitelhoff/Müller 2005; Hasenclever 2001). So könnte beispielsweise eine ungewöhnlich hohe Kooperationsdichte ein Hinweis auf Vertrauen sein, wenn sich die Qualität der Zusammenarbeit anders nicht erklären ließe. Solche kontrafaktischen Szenarien bleiben aber in den Sozialwissenschaften störanfällig, da wir gute Gründe zu der Annahme haben, dass konkrete Handlungsentscheidungen in aller Regel überdeterminiert sind und sich durchaus aus unterschiedlichen metatheoretischen Perspektiven interpretieren lassen (vgl. Hanrieder 2008: 176). Deshalb führt kein Weg daran vorbei, zunächst zu klären, wer in den internationalen Beziehungen sinnvollerweise als Subjekt von Vertrauen identifiziert werden kann, um sich dann zu überlegen, was geeignete Indikatoren sein könnten, um Vertrauen in den internationalen Beziehungen in nachvollziehbarer Art und Weise anzuzeigen.

\subsection{Wer vertraut wem?}

Innerhalb der Forschung zu Vertrauen zwischen Organisationen wird generell davon ausgegangen, dass es sich sowohl beim Vertrauensgeber als auch beim Vertrauensnehmer um individuelle oder kollektive Akteure handeln kann (Currall/ Inkpen 2006: 237; Fulmer/Gelfand 2012: 1170; Janowicz/Noorderhaven 2006; Sydow 2006) ${ }^{44}$ Entsprechend lassen sich in der Literatur vier Arten von Vertrauen unterscheiden: Vertrauen zwischen Individuen (interpersonales Vertrauen), gruppenspezifisches Vertrauen von Individuen, personenspezifischen Vertrauen von Gruppen und Vertrauen zwischen Gruppen (interorganisationelles Vertrauen).

44 Siehe eine ähnliche Akteurskonzeption bei Govier und Verwoerd (2002: 187) und Bart Nooteboom (2006). 
Tabelle 2: Mögliche Akteurskonstellationen

\begin{tabular}{|c|c|c|}
\hline Vertrauensnehmer & Individuum & Kollektiv \\
\hline Individuum & $\begin{array}{c}\text { Vertrauen zwischen } \\
\text { Individuen }\end{array}$ & $\begin{array}{c}\text { Gruppenspezifisches Vertrauen } \\
\text { von Individuen }\end{array}$ \\
\hline Kollektiv & $\begin{array}{c}\text { Personenspezifisches Vertrauen } \\
\text { von Gruppen }\end{array}$ & $\begin{array}{c}\text { Vertrauen zwischen } \\
\text { Gruppen }\end{array}$ \\
\hline
\end{tabular}

Interpersonales Vertrauen gilt als die Grundform des Vertrauens (Janowicz/Noorderhaven 2006: 270; Offe 2001: 253; Zaheer et al. 1998: 143). Vertrauensvolle Personen erwarten von ihren Partnern, dass sie willens und fähig sind, riskante Vorleistungen angemessen zu erwidern. Vertrauen in der internationalen Politik bedeutet dann zunächst und zuerst, dass die außenpolitisch Verantwortlichen den Zusagen ihrer Partner Glauben schenken: Ihren Worten werden Taten folgen, und diese Taten werden geeignet sein, die Vereinbarungen so zu verwirklichen, dass die Interessen der Partner angemessen berücksichtigt werden. In diesem Sinne spricht einiges dafür, dass sich François Mitterrand und Helmut Kohl als aufrichtige und durchsetzungsstarke Politiker schätzten. Bei allen inhaltlichen Gegensätzen gingen sie davon aus, dass der andere sie nicht bewusst hintergehen würde. In ähnlicher Weise müssen sich Menachem Begin und Anwar Sadat oder Raùl Alfonsin und José Sarnay aufeinander verlassen haben, als sie sich daran machten, die ägyptische-israelischen bzw. die argentinisch-brasilianischen Beziehungen von Konfrontation auf Kooperation umzustellen. Dabei blendeten sie nicht nur die Möglichkeit opportunistischen Verhaltens ihrer Partner aus und unterstellten ihnen ein starkes Interesse an dauerhafter Kooperation, sondern sie gingen auch davon aus, dass sie ihre Verabredungen innenpolitisch durchsetzen konnten. ${ }^{45}$

Gruppenspezifisches Vertrauen von Individuen liegt vor, wenn sich Akteure ohne größere Not auf Fremde verlassen. Letztere werden dabei als Mitglieder vertrauenswürdiger Bevölkerungsgruppen oder als Repräsentanten vertrauenswürdiger Organisationen wahrgenommen. Im ersten Fall sprechen Carl Offe (2001: 271) und Jörg Sydow (2006: 285) von kategorialem Vertrauen. Es geht dann nicht darum, dass der Vertrauensgeber den Vertrauensnehmer persönlich kennt und vertraut, sondern dass er ihn als Angehörigen einer sozialen Kategorie erkennt und vertraut - also beispielsweise als Vertreter einer Ethnie, Religion oder auch Partei, der vom Ver-

45 Wenn Vertrauen ausschließlich auf einer solchen interindividuellen Ebene stattfindet, dann müsste zwischenstaatliches Vertrauen zum einen mit wechselnden Politiker-Generationen variieren. Zum anderen müsste letztendlich bezweifelt werden, ob langfristiges Vertrauen zwischen Staaten überhaupt möglich ist, da Vertrauensgeber immer damit rechnen müssen, dass der als vertrauenswürdig eingeschätzte Interaktionspartner sein Amt verliert und somit die Basis der Vertrauensbeziehung wegfällt. Vertrauen, welches ganzen Gruppen entgegen gebracht wird, könnte hingegen lange Vertrauenshorizonte erklären. Andere Ansätze, die von einer diskursiven Verankerung von Vertrauenserwartungen ausgehen sind dazu ebenso fähig, wenn davon ausgegangen wird, dass ein solcher Diskurs über mehrere Generationen vererbt wird. 
trauensgeber insgesamt Zuverlässigkeit und Wohlwollen gegenüber den eigenen Interessen unterstellt wird. ${ }^{46}$ So zeigen beispielsweise Umfragen des Eurobarometers, dass Schweden, Niederländer, Luxemburger und Dänen innerhalb der EU als besonders vertrauenswürdig eingeschätzt werden, während Italienern und Griechen deutlich skeptischer begegnet wird (Delhey 2004: 24). ${ }^{47}$ Im zweiten Fall von gruppenspezifischem Vertrauen ist der Begriff des Systemvertrauens einschlägig (Giddens 1990; Luhmann 2009; Möllering 2006c). Jetzt geht es um Vertrauen in das Funktionieren von Organisationen wie das Polizei- oder Rechtswesen. Hier kann beispielsweise die Zuverlässigkeit der Polizei von den Bürgern und Bürgerinnen unterschiedlich eingeschätzt werden. Entsprechend stark oder schwach ist dann auch die Erwartung in das vertrauenswürdige Handeln von Polizisten ausgeprägt (Janowicz/Noorderhaven 2006: 268; Nooteboom 2006: 259; Sydow 2006: 382). ${ }^{48}$

Wie Akbar Zaheer, Bill McEvily und Vincenzo Perrone (1998: 141) feststellen, können kollektive Akteure genauso wenig vertrauen, wie sie denken oder fühlen können: »It is individuals as members of organizations, rather than the organizations themselves, who trust«. Gleichwohl lassen sich zwischen kollektiven Akteuren mehr oder weniger vertrauensvolle Beziehungen unterscheiden (Bachmann/Inkpen 2011; Möllering 2006b; Sydow 2006). Diese Beziehungen werden durch Diskurse konstituiert, in denen kollektive Wahrnehmungen in Form von gruppenspezifischen Freund- und Feindbildern transportiert werden und die für die Kooperationsbereitschaft der zuständigen Entscheidungsträger relevant sind (Koeszegi 2004: 647-648; Zaheer et al. 1998: 143). ${ }^{49}$ Empirisch lässt sich diese Art des Vertrauens auf unterschiedliche Art und Weise abgreifen. Einen Weg stellen wiederum Umfragen dar, in denen gezeigt werden kann, dass eine Mehrheit von Gruppenmitgliedern einer anderen Staatsbevölkerung vertraut. So wird in Deutschland und Frankreich von Meinungsforschungsinstituten wie Infratest Dimap, dem Pew Research Center oder Sofres regelmäßig erhoben, welchem anderen Land die Bürger und Bürgerinnen vertrauen. Dabei zeigt sich, dass die deutsch-französischen Beziehungen sehr gut

46 Wie oben ausgeführt wurde, können Gruppenkategorisierungen als Basis für Aufhebungsprozesse betrachtet werden. Besonders stark ist deshalb eine gruppenspezifische Vertrauenserwartung, wenn die Gruppe als Teil der eigenen Identitätsgruppe interpretiert wird (Lewicki/Bunker 1995; Uslaner 2002: 28).

47 Eine Studie von Bill McEvily und Akbar Zaheer (2006) zeigt übrigens, dass Individuen Vertrauenserfahrungen mit Einzelmitgliedern einer Gruppe auf die gesamte Gruppe verallgemeinern.

48 Während also einzelnen Polizisten im Fall von interaktions-basiertem Vertrauen deshalb vertraut wird, weil sie persönlich bekannt sind, begegnet ihnen der Vertrauensgeber im Fall von kategorien-basiertem oder institutionen-basiertem Vertrauen als Repräsentant einer vertrauenswürdigen Gruppe oder Organisation. In den letzten beiden Fällen ist die Gruppe oder Organisation das Objekt des Vertrauens (Möllering 2006b: 355) und trägt das Vertrauen in ihre Repräsentanten, die für den Vertrauensgeber durchaus Fremde bleiben können (Bachmann/Inkpen 2011: 282).

49 In dem Augenblick, in dem allerdings kollektiven Akteuren Interessen unterstellt werden, wie dies gerade in den Internationalen Beziehungen oft der Fall ist, kann es auch Sinn machen, vom Vertrauen kollektiver Akteure zu sprechen. Dann gilt: »An organization's trust is signified by a decision to engage in action that allows its fate to be determined by another person, group or organization« (Currall/Inkpen 2006: 236). Ähnlich argumentieren Nooteboom (2006: 249) und Sydow (2006: 381). 
abschneiden, während die deutsch-chinesischen oder die französisch-russischen Beziehungen deutlich schlechtere Werte erhalten. Ein anderes Verfahren zur Erfassung interkollektiver Vertrauensbeziehungen stellt die Befragung sogenannter boundary spanners (»Grenzgänger«) dar (Sydow 2006: 381; Zaheer/Harris 2006: 185). Hierbei handelt es sich um Personen mit guten Kontakten zu einer fremden Gruppe, deren Meinung in der eigenen Gruppe Gewicht hat bzw. als Substitut für die Gruppenerwartung betrachtet werden kann. Deshalb wird in der Literatur erwartet, dass boundary spanners über Vertrauensbeziehungen zwischen Gruppen zuverlässig Auskunft geben können.

Die vierte Vertrauensform betrifft wieder die Beziehungen zwischen kollektiven und individuellen Akteuren. Allerdings haben wir es jetzt mit Gruppen als Vertrauensgebern und Individuen als Vertrauensnehmern zu tun. Deshalb spielen auch bei dieser Vertrauensform Diskurse eine zentrale Rolle, in denen die Wertschätzung von Gruppen für Personen sichtbar wird. So hatten beispielsweise 87 Prozent der Deutschen im März 2012 wenig oder gar kein Vertrauen in den russischen Präsidenten Wladimir Putin (Infratest Dimap 2012).

In der Literatur zur Rolle von Vertrauen in Politik und Gesellschaft wird davon ausgegangen, dass alle vier Formen von Vertrauen für die Einschätzung konkreter Vertrauensverhältnisse relevant sind. ${ }^{50}$ Personen setzen mehr oder weniger großes Vertrauen in andere Personen und Gruppen. Sie lassen sich dabei von ihren individuellen Wahrnehmungen und Einschätzungen leiten, die selbst wiederum nachhaltig von Diskursen und den dort transportierten kollektiven Wahrnehmungen und Einschätzungen zur Vertrauenswürdigkeit von Personen und Gruppen beeinflusst werden. Innerhalb dieses mehrdimensionalen Vertrauenssystems kommt der interpersonellen Ebene zunächst ein analytischer Primat zu (Janowicz/Noorderhaven 2006; Schweer 2012: 112; Zaheer et al. 1998). Das gilt vor allem für den Aufbau von Vertrauensverhältnissen in schwach institutionalisierten Kontexten (Schweer 2012: 112; Zaheer/Harris 2006: 185). Gleichzeitig zeigen Studien aber auch, dass konkretes Misstrauen zwischen Personen durch Vertrauen in Institutionen kompensiert werden kann. So lässt sich nach Möllering (2006c: 365, 373) oder Sydow (2006: 381) immer wieder beobachten, dass sich ein Vertrauensgeber zu einer Vertrauenshandlung entschließt, weil er überzeugt ist, dass die aus seiner Sicht wenig vertrauenswürdige Person, mit der er es unmittelbar zu tun hat, in eine vertrauenswürdige Organisation eingebunden ist, die ihre Repräsentanten hinreichend kontrolliert.

Schließlich ist davon auszugehen, dass Vertrauensdiskurse für die Vertrauensbereitschaft von Vertrauensgebern eine zentrale Rolle spielen. Deshalb wird Vertrauen von Möllering (2006c: 365) auch als embedded agency begriffen. Je nachdem wie stark diese Vertrauensdiskurse ausgeprägt sind, kann es sogar Sinn machen, kollektive Akteure so zu behandeln, als ob sie vertrauen könnten. Dies gilt vor allem für Fälle, in denen kollektive Akteure in hoch institutionalisierten Kontexten

50 Currall/Inkpen (2006: 240); Fulmer/Glefand (2012: 11-68); Lewis/Weigert (1985: 974); Schweer (2012: 108). 
eingebunden sind, in denen Vertrauen quasi als Norm erscheint (vgl. auch Uslaner (2002: Kap.2) für die normative Basis von Vertrauen). Die deutsch-französischen Beziehungen wären ein Beispiel. Umgekehrt kann es natürlich auch sein, dass Feindseligkeiten zwischen Gruppen oder Staaten so massiv sind, dass letztere als kollektive Akteure behandelt werden können, die unter den Bedingungen eines ausgeprägten Sicherheitsdilemmas ihre mehr oder weniger rationalen Entscheidungen treffen.

\subsection{Zwei Indikatoren für Vertrauen zwischen Staaten}

Im Folgenden wollen wir eine Möglichkeit der Operationalisierung von zwischenstaatlichen Vertrauensbeziehungen skizzieren. Dabei gehen wir davon aus, dass nicht nur das Vertrauensverhältnis zwischen zwei Staaten politikfeldspezifisch variieren kann, sondern auch mögliche Indikatoren an die Eigenschaften unterschiedlicher Interaktionsfelder angepasst werden müssen. Deshalb begrenzen wir uns im Folgenden auf das Politikfeld Sicherheit. Dabei orientieren wir uns an Hoffman (2002) und Rathbun (2011b), die es bislang als Einzige versucht haben, strukturierte Indikatoren für zwischenstaatliches Vertrauen zu entwickeln. Aus akteurstheoretischer Sicht fokussieren wir Vertrauenserwartungen von individuellen Entscheidungsträgern bzw. von Gruppen von Entscheidungsträgern. Denn letztendlich stehen sie in der Verantwortung und entscheiden, ob sie dem Interaktionspartner vertrauen wollen oder ihn kontrollieren müssen. ${ }^{51}$ Dementsprechend sehen wir die Möglichkeit, für die empirische Feststellung von Vertrauensbeziehungen auf unsere konzeptionellen Überlegungen zum Komplementärverhältnis von Vertrauen, Information und Kontrolle zurückzugreifen.

Kontrollmechanismen spielen in den internationalen Beziehungen unbestreitbar eine wichtige Rolle. Selbst im Rahmen gut etablierter Sicherheitsgemeinschaften kommt es immer wieder zu Irritationen, die durch die Weiterentwicklung gemeinsamer Institutionen bearbeitet werden müssen. Dies zeigen nicht zuletzt die verschiedenen Institutionalisierungsschübe in den deutsch-französischen Beziehungen nach 1960. Ebenso gehört die Einholung von Informationen - auch über befreundete Staaten - zur täglichen Aufgabe der Politik. Gleichzeitig müssen aber Kontrolle und Informationsgewinnung nicht alles sein. Für die Analyse konkreter zwischenstaatlicher Vertrauensbeziehungen stellt sich deshalb die Frage nach dem Mischungsverhältnis von Aufhebung, Kalkül und Kontrolle hinsichtlich ihrer kooperationsermöglichenden Wirkung.

Wie viele andere Theoretiker gehen auch wir davon aus, dass sich zunehmendes Vertrauen im abnehmenden Gebrauch von Kontrollmechanismen niederschlagen sollte (Offe 2001: 248). Zudem unterstellen wir dem Vertrauensgeber, dass er abwägt, ob er stark genug vertraut, um auf Informationseinholung und Kontrolle ver-

51 Die Frage, wem dieses Vertrauen entgegengebracht wird, ist für unsere Operationalisierungsskizze unerheblich. 
zichten zu können, oder aber ob das vorhandene Vertrauen hierfür nicht ausreicht. Mit wachsendem Vertrauen sollten Staaten somit weniger Ressourcen für Informationsbeschaffungsmaßnahmen aufbringen. Ministerielle, nachrichtendienstliche und militärische Einheiten, die sich mit spezifischen bilateralen Sicherheitsbeziehungen befassen, sollten schwächer besetzt werden. Zudem könnte angenommen werden, dass vertrauende Staaten auf die Errichtung von Kontrollmechanismen verzichten, zum Beispiel auf teure militärische Abschreckungskapazitäten, auf institutionalisierte Formen von Sicherheitskooperation bzw. auf die Möglichkeit intrusiver Verifikationsmechanismen. ${ }^{52}$ Eine solche Erfassung von Vertrauensbeziehungen kann allerdings nur unter bestimmten Bedingungen gelingen: Erstens muss sichergestellt werden, dass die bloße Möglichkeit militärischer Aggression besteht. Vertrauen muss aus objektiver Sicht ein Wagnis darstellen: Deutschland kann Frankreich sicherheitspolitisch vertrauen, aber nicht Neuseeland. Zweitens muss darauf geachtet werden, dass der Nexus zwischen Verifikation und Vertrauen in der Realität komplizierter ist, da, wie oben bereits angedeutet, die Akzeptanz von Verifikation schon ein Mindestmaß an Vertrauen voraussetzen könnte. Drittens kann von der bloßen Existenz von Verifikationsmaßnahmen nicht auf fehlendes Vertrauen geschlossen werden: Auf ihre Nutzung kommt es an. Zum Beispiel sind Deutschland und Frankreich Mitglied im Open-Skies-Verifikationsregime, jedoch nutzt keines der beiden Länder seine Überflugrechte zur Kontrolle des jeweils anderen. Zudem muss beachtet werden, dass neben den oben genannten direkt beobachtbaren Kontroll- und Informierungsaktivitäten weitere, weniger direkt beobachtbare Mechanismen existieren, die positive Kooperationserwartungen entstehen lassen können (etwa Kooperation aufgrund von Reputationssorgen, starker wirtschaftlicher Interdependenzen oder Kooperation aufgrund von audience costs, die demokratische Entscheidungsträger zu tragen haben, wenn sie ihr Wahlversprechen über kooperatives Verhalten nicht einhalten). Es zeigt sich also, dass kontextsensible Messung unbedingt wichtig ist (Adcock/Collier 2001: 534). Für eine empirische Analyse empfiehlt es sich deshalb auch, solche Drittfaktoren konstant zu halten.

Da dies jedoch nicht immer möglich erscheint, schlagen wir zur Absicherung der Validität der Vertrauensmessung einen Komplementärindikator vor, der auf die Aussagen entweder von politischen Entscheidungsträgern oder aber im gesamtge-

52 Wenn man von der Möglichkeit eines Mehr oder Weniger an Vertrauen in zwischenstaatlichen Beziehungen ausgeht, stellt sich natürlich die Frage nach der Skalierung von Vertrauen und, damit verbunden, nach der Messung unterschiedlicher Vertrauensniveaus. Hier könnte argumentiert werden, dass sich unterschiedlich starkes Vertrauen in der unterschiedlich kombinierbaren Anwendung möglicher Komplementärmechanismen niederschlagen sollte. Der Aspekt der logischen und validen Skalierung soll an dieser Stelle jedoch nicht weiter ausgeführt werden. 
sellschaftlichen Diskurs abzielt. ${ }^{53}$ Ein solcher zweiter Indikator ermöglicht es, durch die Analyse von verbalen und schriftlichen Aussagen Vertrauenserwartungen zu erheben. So sollten sich Vertrauenserwartungen erstens in der gegenseitigen Zusprechung bestimmter vertrauenswürdiger Eigenschaften widerspiegeln (Loyalität, Solidarität, Freundschaft, Verbundenheit). ${ }^{54}$ Zweitens sollten vertrauensvolle Beziehungen durch Akteure mit eindeutigen, emotionalen Attributen versehen werden, die auf Identifikation und Verbundenheit hindeuten. Drittens sollte allein die Möglichkeit vertrauensunwürdigen Verhaltens durch den Anderen unbeachtet bleiben. Und schließlich sollte die eigene Kooperationsbereitschaft durch die vertrauensvollen Beziehungen selbst begründet werden.

Unser Operationalisierungsvorschlag kann an dieser Stelle nur als grobe Skizze verstanden werden, die durch konkrete Messanweisungen und Regeln spezifiziert werden muss. Dabei sehen wir die Kombination der beiden Indikatoren als unerlässlich an, da nur auf diese Weise ihre jeweiligen Schwächen ausgeglichen werden können. Zudem erlaubt die kombinierte Anwendung eine Absicherung der Validität der Vertrauensmessung, wenn die Ergebnisse der Analyse von Aussagen und Kooperationsausgestaltung im vertrauenstheoretisch $\mathrm{zu}$ erwartenden Verhältnis miteinander kovariieren. Die von uns propagierte Messstrategie stellt somit hohe Anforderungen an die benötigten Daten hinsichtlich ihrer Detailtiefe und Fülle. Eine Quantifizierung von Vertrauensindikatoren scheint deshalb noch in weiter Ferne, weshalb wir als Anwendungsgebiet solcher Indikatoren vornehmlich vergleichende Fallanalysen und Prozessanalysen sehen.

\section{Konklusion}

In dem vorliegenden Aufsatz haben wir einen Überblick zum Thema Vertrauen als Forschungsgegenstand in den Internationalen Beziehungen gegeben. Vertrauen ist ein oft bemühter Begriff - vor allem in der politischen Praxis aber zunehmend auch in der IB-Forschung. Allerdings haben sich bislang nur wenige mit der Frage auseinander gesetzt, was Vertrauen ist bzw. in der politischen Realität überhaupt sein kann. Deshalb haben wir zunächst die rationalistische und die von uns als aufhebungsbasiert benannte Vertrauensontologie diskutiert und dabei argumentiert, dass ein rationalistischer Zugang zu dem Phänomen des Vertrauens unterkomplex ist. Möchte man Vertrauen als realweltliches und ontologisch eigenständiges Phäno-

53 Ein weiterer guter Grund für einen solchen Komplementärindikator besteht in der Möglichkeit, dass individuell existierende Vertrauenserwartungen sich aufgrund widriger struktureller Gegebenheiten nicht notwendigerweise in Handlungen niederschlagen müssen. So könnte ein tatsächlich vertrauender Entscheidungsträger etwa aufgrund der Gesetzeslage gezwungen sein, auf vertrauensvolle Handlungen zu verzichten. Für die Erfassung der individuellen Vertrauenserwartung würde ein strikt handlungsbasierter Indikator in diesem Fall ein falsch-negatives Ergebnis liefern.

54 Das Problem strategischer Sprechakte und weiterer Fallstrike bei solchen Inhaltsanalysen sind immer ein Problem und müssen freilich berücksichtigt werden. 
men begreifen, empfehlen wir die Anwendung eines aufhebungsbasierten Vertrauenskonzepts.

Wegen seiner Nähe zu etablierten Modellen der strategischen Entscheidung ist rationalistisches Vertrauen nicht mehr als alter Wein in neuen Schläuchen. Deshalb verwundert es auch nicht, wenn es sich implizit in den meisten rationalen Erklärungsmodellen zu internationaler Kooperation wiederfinden lässt. Explizit beschäftigt sich Kydd (2000; 2005) mit zwischenstaatlichem Vertrauen aus rationalistischer Perspektive. Erste Studien, die versuchen ein explizit aufhebungsbasiertes Konzept von Vertrauen für die Analyse internationaler Politik zu entwickeln, liegen zwar vor. Während ihre konzeptionellen Leistungen zwar durchaus überzeugen können, tragen die empirischen Befunde bislang noch nicht die hohen theoretischen Erwartungen. Deshalb schlagen wir zum einen akteurstheoretische Fundierungen für zwischenstaatliches Vertrauen vor, zum anderen bieten wir einen Ansatz zur Operationalisierung von Vertrauen.

Abschließend wollen wir noch auf eine, aus vertrauensskeptischer Perspektive oftmals angebrachte Frage über die mögliche dark side von Vertrauen hinweisen: Experimentelle Studien zeigen, dass die Bildung starker Vertrauensbeziehungen verheerende in-group-out-group-Dynamiken auslösen können. In-Group-Vertrauen zwischen Staaten kann dann leicht mit Misstrauen gegenüber anderen Staaten einhergehen und eine erhöhten Bereitschaft hervorrufen, Vertretern der out-group Schaden zuzufügen (Ostrom 2002: 45). In diesem Sinne muss ein bedingungsloser Vertrauensoptimismus sicherlich relativiert und damit verbunden die Frage gestellt werden, wie Vertrauensbildung so gestaltet werden kann, dass destruktive und misstrauensgenerierende Wirkungen gegenüber Dritten vermieden werden können.

\section{Literatur}

Adcock, Robert/Collier, David 2001: Measurement Validity: A Shared Standard for Qualitative and Quantitative Research, in: The American Political Science Review 95: 3, 529-546.

Adler, Emanuel 1997: Imagined (Security) Communities: Cognitive Regions in International Relations, in: Millennium: Journal of International Studies 26: 2, 249-277.

Adler, Emanuel/Barnett, Michael 1998a: A Framework for the Study of Security Communities, in: Adler, Emanuel/Barnett, Michael (Hrsg.): Security Communities, Cambridge, 29-66.

Adler, Emanuel/Barnett, Michael 1998b: Security Communities in Theoretical Perspective, in: Adler, Emanuel/Barnett, Michael (Hrsg.): Security Communities, Cambridge, 3-28.

Adler, Emanuel/Greve, Patricia 2009: When Security Community Meets Balance of Power: Overlapping Regional Mechanisms of Security Governance, in: Review of International Studies 35: S1, 59-84.

Alexander, Michele G./Brewer, Marilynn B./Herrmann, Richard 1999: Images and Affect: A Functional Analysis of Out-Group Stereotypes, in: Journal of Personality and Social Psychology 77: 1, 78-93.

Axelrod, Robert 1984: The Evolution of Cooperation, New York, NY. 
Bachmann, Reinhard/Inkpen, Andrew C. 2011: Understanding Institutional-Based Trust Building Processes in Inter-Organization Relationships, in: Organizational Studies 32: 2, 281-301.

Barbalet, Jack 1996: Social Emotions: Confidence, Trust and Loyalty, in: International Journal of Sociology and Social Policy 16: 9/10, 75-96.

Barbalet, Jack 2009: A Characterization of Trust, and its Consequences, in: Theory and Society 38: 4, 367-382.

Barnett, Michael/Adler, Emanuel 1998: Studying Security Communities in Theory, Comparison and History, in: Adler, Emanuel/Barnett, Michael (Hrsg.): Security Communities, Cambridge, 413-441.

Baron, Reuben M. 2008: Reconciliation, Trust, and Cooperation: Using Bottom-Up and TopDown Strategies to Achieve Peace in the Israeli-Palestinian Conflict, in: Nadler, Arie/ Malloy, Thomas/Fischer, Jeffery (Hrsg.): The Social Psychology of Intergroup Reconciliation, New York, NY, 275-298.

Becker, Lawrence C. 1996: Trust as Non-Cognitive Security about Motives, in: Ethics 107: 1, 43-61.

Beckert, Jens 1996: Was ist soziologisch an der Wirtschaftssoziologie? Ungewißheit und die Einbettung wirtschaftlichen Handelns, in: Zeitschrift für Soziologie 25: 2, 125-146.

Bengtsson, Rikard 2000: The Cognitive Dimension of Stable Peace, in: Kacowicz, Arie M./ Bar-Siman-Tov, Yaacov/Jerneck, Magnus (Hrsg.): Stable Peace among Nations, Lanham, MD, 92-107.

Bijlsma-Frankema, Katinka/Costa, Ana C. 2005: Understanding the Trust-Control Nexus, in: International Sociology 20: 3, 259-282.

Bilali, Rezarta 2010: Assessing the Internal Validity of Image Theory in the Context of Turkey-U.S. Relations, in: Political Psychology 31: 2, 275-303.

Binmore, Ken 2009: Rational Decisions, Princeton, NJ.

Blum, Gesa 2003: Vertrauensarbeit. Deutsch-französische Beziehungen nach 1945, in: Frevert, Ute (Hrsg.): Vertrauen. Historische Annäherungen, Göttingen, 365-393.

Booth, Ken/Wheeler, Nicholas J. 2008: The Security Dilemma: Fear, Cooperation, and Trust in World Politics, Houndmills.

Braun, Norman 1992: Altruismus, Moralität und Vertrauen, in: Analyse \& Kritik 14, 177-186.

Brewer, Marilynn B./Alexander, Michele G. 2002: Intergroup Emotions and Images, in: Mackie, Diane M./Smith, Eliot R. (Hrsg.): From Prejudice to Intergroup Emotions: Differentiated Reactions to Social Groups, 209-226.

Brewer, Paul R./Gross, Kimberly/Aday, Sean/Willnat, Lars 2004: International Trust and Public Opinion about World Affairs, in: American Journal of Political Science 48: 1, 93-109.

Coleman, James S. 1994: Foundations of Social Theory, Cambridge, MA.

Cook, Karen S./Cooper, Robin M. 2003: Experimental Studies of Cooperation, Trust, and Social Exchange, in: Ostrom, Elinor/Walker, James (Hrsg.): Trust \& Reciprocity: Interdisciplinary Lessons from Experimental Research, New York, NY, 209-244.

Cook, Karen S./Yamagishi, Toshio/Cheshire, Coye/Cooper, Robin/Matsuda, Masafumi/ Mashima, Rie 2005: Trust Building via Risk Taking: A Cross-Societal Experiment, in: Social Psychology Quarterly 68: 2, 121-142.

Currall, Steven C.Inkpen, Andrew C. 2006: On the Complexity of Organizational Trust: A Multi-Level Co-Evolutionary Perspective and Guidelines for Future Research, in: Bachmann, Reinhard/Zaheer, Akbar (Hrsg.): Handbook of Trust Research, Cheltenham, 235-246.

Das, T. K./Teng, Bing-Sheng 2001: Trust, Control, and Risk in Strategic Alliances: An Integrated Framework, in: Organization Studies 22: 2, 251-283.

Das, T. K./Teng, Bing-Sheng 2004: The Risk-Based View of Trust: A Conceptual Framework, in: Journal of Business and Psychology 19: 1, 85-116. 
Davidson, Paul 1991: Is Probability Theory Relevant for Uncertainty? A Post-Keynesian Perspective, in: Journal of Economic Perspectives 5: 1, 129-143.

Deitelhoff, Nicole/Müller, Harald 2005: Theoretical Paradise - Empirically Lost? Arguing with Habermas, in: Review of International Studies 31: 1, 167-179.

Delhey, Jan 2004: Nationales und transnationales Vertrauen in der Europäischen Union, in: Leviathan 32: 1, 15-44.

Deutsch, Morton 1958: Trust and Suspicion, in: Journal of Conflict Resolution 2: 4, 265-279.

Deutsch, Morton 1962: Cooperation and Trust: Some Theoretical Notes, in: Jones, Marshall R. (Hrsg.): Nebraska Symposium on Motivation, Lincoln, NE, 275-319.

Dovidio, John F./Gaertner, Samuel L./John, Melissa-Sue/Halabi, Samer/Saguy, Tamar/Pearson, Adam R./Riek, Blake M. 2008: Majority and Minority Perspectives in Intergroup Relations: The Role of Contact, Group Representations, Threat, and Trust in Intergroup Conflict and Reconciliation, in: Nadler, Arie/Malloy, Thomas/Fischer, Jeffery (Hrsg.): The Social Psychology of Intergroup Reconciliation, New York, NY, 227-273.

Fiedler, Martin 2001: Vertrauen ist gut, Kontrolle ist teuer. Vertrauen als Schlüsselkategorie wirtschaftlichen Handelns, in: Geschichte und Gesellschaft - Zeitschrift für historische Sozialwissenschaft 27: 4, 576-592.

Fischer, Lorenz/Wiswede, Günter 2009: Grundlagen der Sozialpsychologie, München.

Flemes, Daniel 2003: Entsteht im südlichen Lateinamerika eine Sicherheitsgemeinschaft? Ausgangslage, Stand und Perspektiven sicherheitspolitischer Kooperation, Hamburg.

Fulmer, C. Ashley/Gelfand, Michael J. 2012: At What Level (and in Whom) We Trust: Trust Across Multiple Organizational Levels, in: Journal of Management 38: 4, 1167-1230.

Giddens, Anthony 1985: The Constitution of Society, Berkeley, CA.

Giddens, Anthony 1990: The Consequences of Modernity, Stanford, CA.

Gilboa, Itzhak/Postlewaite, Andrew/Schmeidler, David 2012: Rationality of Belief or Why Savage's Axioms are Neither Necessary nor Sufficient for Rationality, in: Synthese 187: 1, 11-31.

Gillespie, Alex 2012: Dialogical Dynamics of Trust and Distrust in the Cuban Missile Crisis, in: Marková, Ivana/Gillespie, Alex (Hrsg.): Trust and Conflict: Representation, Culture and Dialogue, New York, NY, 139-155.

Glaser, Charles L. 1994: Realists as Optimists: Cooperation as Self-Help, in: International Security 19: 3, 50-90.

Govier, Trudy/Verwoerd, Wilhelm 2002: Trust and the Problem of National Reconciliation, in: Philosophy of the Social Sciences 32: 2, 178-205.

Hanrieder, Tine 2008: Moralische Argumente in den Internationalen Beziehungen. Grenzen einer verständigungstheoretischen »Erklärung« moralischer Debatten, in: Zeitschrift für Internationale Beziehungen 15: 2, 161-186.

Hardin, Russell 2002: Trust \& Trustworthiness, New York, NY.

Hardin, Russell 2006: Trust, Cambridge, MA.

Hasenclever, Andreas 2001: Die Macht der Moral in der internationalen Politik. Militärische Interventionen westlicher Staaten in Somalia, Ruanda und Bosnien-Herzegowina, Frankfurt a. M.

Hayashi, Nahoko/Ostrom, Elinor/Walker, James/Yamagishi, Toshio 1999: Reciprocity, Trust and the Sense of Control: A Cross-Societal Study, in: Rationality and Society 11: 1, 27-46.

Herborth, Benjamin 2007: Verständigung verstehen. Anmerkungen zur ZIB-Debatte, in: Niesen, Peter/Herborth, Benjamin (Hrsg.): Anarchie der kommunikativen Freiheit. Jürgen Habermas und die Theorie der internationalen Politik., Frankfurt a. M., 147-172.

Herrmann, Richard 1998: The Empirical Challenge of the Cognitive Revolution: A Strategy for Drawing Inferences about Perceptions, in: International Studies Quarterly 32: 2, 175-203. 
Herrmann, Richard K. 2003: Image Theory and Strategic Interaction in International Relations, in: Sears, David O./Huddy, Leonie/Jervis, Robert (Hrsg.): Oxford Handbook of Political Psychology, Oxford, 253-284.

Herrmann, Richard K./Voss, James F./Schooler, Tonya Y. E./Ciarrochi, Joseph 1997: Images in International Relations: An Experimental Test of Cognitive Schemata, in: International Studies Quarterly 41: 3, 403-433.

Hewstone, Miles/Jaspars, Jos/Lalljee, Mansur 1982: Social Representations, Social Attribution and Social Identity: The Intergroup Images of >Public $<$ and >Comprehensive Schoolboys, in: European Journal of Social Psychology 12: 3, 241-269.

Hewstone, Miles/Kenworthy, Jared B./Cairns, Ed/Tausch, Nicole/Hughes, Joanne/Tam, Tania/Voci, Alberto/Hecker, Ulrich von/Pinder, Catherine 2008: Stepping Stones to Reconciliation in Northern Ireland: Intergroup Contact, Forgiveness, and Trust, in: Nadler, Arie/Malloy, Thomas/Fischer, Jeffery (Hrsg.): The Social Psychology of Intergroup Reconciliation, New York, NY, 199-226.

Hoffman, Aaron M. 2002: A Conceptualization of Trust in International Relations, in: European Journal of International Relations 8: 3, 375-401.

Hoffman, Aaron M. 2006: Building Trust: Overcoming Suspicion in International Conflict, Albany, NY.

Hoffman, Aaron M. 2007: The Structural Causes of Trusting Relationships: Why Rivals Do Not Overcome Suspicion Step by Step, in: Political Science Quarterly 122: 2, 287-312.

Hogg, Michael A. 2000: Subjective Uncertainty Reduction through Self-Categorization: A Motivational Theory of Social Identity Processes, in: European Review of Social Psychology 11: 1, 223-255.

Hogg, Michael A./Sherman, David K./Dierselhuis, Joel/Maitner, Angela T./Moffitt, Graham 2007: Uncertainty, Entitativity, and Group Identification, in: Journal of Experimental Social Psychology 43: 1, 135-142.

Infratest Dimap 2012: ARD-DeutschlandTREND, in: http://www.infratest-dimap.de/umfragen-analysen/bundesweit/ard-deutschlandtrend/2012/maerz/; 1.10.2013.

Janowicz, Martyna/Noorderhaven, Niels 2006: Levels of Inter-Organizational Trust: Conceptualization and Measurement, in: Bachmann, Reinhard/Zaheer, Akbar (Hrsg.): Handbook of Trust Research, Cheltenham, 264-279.

Jervis, Robert 1976: Perception and Misperception in International Politics, Princeton, NJ.

Jones, Karen 1996: Trust as an Affective Attitude, in: Ethics 107: 1, 4-25.

Jones, Peter 2008: South Asia: Is a Regional Security Community Possible?, in: South Asian Survey 15: 2, 183-193.

Jönsson, Christer 1993: Cognitive Factors in Regime Dynamics, in: Rittberger, Volker (Hrsg.): Regime Theory and International Relations, Oxford, 202-222.

Kacowicz, Arie M. 2000: Stable Peace in South America: The ABC Triangle, 1979-1999, in: Kacowicz, Arie M./Bar-Siman-Tov, Yaacov/Jerneck, Magnus (Hrsg.): Stable Peace among Nations, Lanham, MD, 200-219.

Kacowicz, Arie M./Bar-Siman-Tov, Yaacov/Jerneck, Magnus (Hrsg.) 2000: Stable Peace among Nations, Lanham, MD.

Kelsey, David/Quiggin, John 1992: Theories of Choice under Ignorance and Uncertainty, in: Journal of Economic Surveys 6: 2, 133-153.

Keohane, Robert O. 1986: Reciprocity in International Relations, in: International Organization 40: 1, 1-27.

Keohane, Robert O. 1993: The Analysis of International Regimes: Towards a European-American Research Programme, in: Rittberger, Volker (Hrsg.): Regime Theory and International Relations, Oxford, 23-45.

Koeszegi, Saobine T. 2004: Trust-Building Strategies in Inter-Organizational Negotiations, in: Journal of Managerial Psychology 19: 6, 640-660.

Kokeyev, Mikhail/Androsov, Andrei 1990: Verification: The Soviet Stance; its Past, Present and Future, New York, NY. 
Koremenos, Barbara/Lipson, Charles/Snidal, Duncan 2001: The Rational Design of International Institutions, in: International Organization 55: 4, 761-799.

Kosfeld, Michael/Heinrichs, Markus/Zak, Paul J./Fischbacher, Urs/Fehr, Ernst 2005: Oxytocin Increases Trust in Humans, in: Nature 435: 7042, 673-676.

Kramer, Roderick M. 1999: Trust and Distrust in Organizations: Emerging Perspectives, Enduring Questions, in: Annual Review of Psychology 50, 569-598.

Kuhlman, D. M./Marshello, Alfred F. 1975: Individual Differences in Game Motivation as Moderators of Preprogrammed Strategy Effects in Prisoners Dilemma, in: Journal of Personality and Social Psychology 32: 5, 922-931.

Kupchan, Charles A. 2010: How Enemies Become Friends: The Sources of Stable Peace, Princeton, NJ.

Kydd, Andrew H. 2000: Trust, Reassurance, and Cooperation, in: International Organization 54: 2, 325-357.

Kydd, Andrew H. 2005: Trust and Mistrust in International Relations, Princeton, NJ.

Lahno, Bernd 2001: On the Emotional Character of Trust, in: Ethical Theory and Moral Practice 4: 2, 171-189.

Lahno, Bernd 2002: Der Begriff des Vertrauens, Paderborn.

Lahno, Bernd 2004: Is Trust the Result of Bayesian Learning?, in: Jahrbuch für Handlungsund Entscheidungstheorie 3, 47-68.

Larson, Deborah W. 1997a: Anatomy of Mistrust: U.S.-Soviet Relations during the Cold War, Ithaca, NY.

Larson, Deborah W. 1997b: Trust and Missed Opportunities in International Relations, in: Political Psychology 18: 3, 701-734.

Lewicki, Roy J./Bunker, Barbara B. 1995: Trust in Relationships: A Model of Development and Decline, in: Bunker, Barbara B./Rubin, Jeffrey Z. (Hrsg.): Conflict, Cooperation and Justice: Essays Inspired by the Work of Morton Deutsch, San Francisco, CA, 133-173.

Lewis, J. David/Weigert, Andrew J. 1985: Trust as a Social Reality, in: Social Forces 63: 4, 967-985.

Lindskold, Svenn 1978: Trust Development, the GRIT Proposal, and the Effects of Conciliatory Acts on Conflict and Cooperation, in: Psychological Bulletin 85: 4, 772-793.

Luhmann, Niklas 2009: Vertrauen. Ein Mechanismus der Reduktion sozialer Komplexität, Stuttgart.

McClintock, Charles G./Liebrand, Wim B. 1988: Role of Interdependence Structure, Individual Value Orientation, and Another's Strategy in Social Decision Making: A Transformational Analysis, in: Journal of Personality and Social Psychology 55: 3, 396-409.

McEvily, Bill/Zaheer, Akbar 2006: Does Trust Still Matter? Research on the Role of Trust in Inter-Organizaitonal Exchange, in: Bachmann, Reinhard/Zaheer, Akbar (Hrsg.): Handbook of Trust Research, Cheltenham, 280-301.

Mearsheimer, John J. 2001: The Tragedy of Great Power Politics, New York, NY.

Mercer, Jonathan 2005: Rationality and Psychology in International Politics, in: International Organization 59: 1, 77-106.

Mercer, Jonathan 2010: Emotional Beliefs, in: International Organization 64: 1, 1-31.

Messick, David M./Kramer, Roderick M. 2003: Trust as a Form of Shallow Morality, in: Cook, Karen S. (Hrsg.): Trust in Society, New York, 89-117.

Michel, Torsten 2012: Time to Get Emotional: Phronetic Reflections on the Concept of Trust in International Relations, in: European Journal of International Relations, in: http:// ejt.sagepub.com/content/early/2012/04/27/1354066111428972.abstract; 27.9.2013.

Möllering, Guido 2001: The Nature of Trust: From Georg Simmel to a Theory of Expectation, Interpretation and Suspension, in: Sociology 35: 2, 401-420.

Möllering, Guido 2005: The Trust/Control Duality: An Integrative Perspective on Positive Expectations of Others, in: International Sociology 20: 3, 283-305.

Möllering, Guido 2006a: Das Aufheben von Ungewissheit als Kern des Vertrauens. Just do it?, in: MPIfG Working Paper 6: 5. 
Möllering, Guido 2006b: Trust, Reason, Routine, Reflexivity, Amsterdam.

Möllering, Guido 2006c: Trust, Institutions, Agency: Towards a Neoinstitutional Theory of Trust, in: Bachmann, Reinhard/Zaheer, Akbar (Hrsg.): Handbook of Trust Research, Cheltenham, 355-376.

Morrow, James D. 1994: Game Theory for Political Scientists, Princeton.

Müller, Harald 2004: Arguing, Bargaining and All That: Communicative Action, Rationalist Theory and the Logic of Appropriateness in International Relations, in: European Journal of International Relations 10: 3, 395-435.

Müller, Harald/Wolff, Jonas 2006: Democratic Peace: Many Data, Little Explanation, in: Geis, Anna/Brock, Lothar/Müller, Harald (Hrsg.): Democratic Wars: Looking at the Dark Side of Democratic Peace, Basingstoke, 41-73.

Nadler, Arie/Liviatan, Ido 2006: Intergroup Reconciliation: Effects of Adversary's Expressions of Empathy, Responsibility, and Recipients' Trust, in: Personality and Social Psychology Bulletin 32: 4, 459-470.

Nadler, Arie/Saguy, Tamar 2004: Reconciliation between Nations: Overcoming Emotional Deterrents to Ending Conflicts between Groups, in: Langholtz, Harvey J./Stout, Chris E. (Hrsg.): The Psychology of Diplomacy, Westport, CT, 29-46.

Neumaier, Maria 2011: Risikomanagement aus dem Unbewussten, in: Erwägen Wissen Ethik 22: 2, 293-295.

Nooteboom, Bart 2006: Forms, Sources and Processes of Trust, in: Bachmann, Reinhard/ Zaheer, Akbar (Hrsg.): Handbook of Trust Research, Cheltenham, 247-263.

Oelsner, Andrea/Vion, Antoine 2011: Special Issue: Friendship in International Relations, in: International Politics 48: 1, 1-9.

Offe, Claus 2001: Wie können wir unseren Mitbürgern vertrauen?, in: Hartmann, Martin/Offe, Claus (Hrsg.): Vertrauen. Die Grundlage des sozialen Zusammenhalts, Frankfurt a. M., 241-294.

Osgood, Charles E. 1962: An Alternative to War or Surrender, Urbana, IL.

Ostrom, Elinor 2002: Toward a Behavioral Theory Linking Trust, Reciprocity and Reputation, in: Ostrom, Elinor/Walker, James (Hrsg.): Trust \& Reciprocity: Interdisciplinary Lessons from Experimental Research, New York, NY, 19-79.

Ostrom, Elinor/Walker, James (Hrsg.) 2002: Trust \& Reciprocity: Interdisciplinary Lessons from Experimental Research, New York, NY.

Pettigrew, Thomas F. 1979: The Ultimate Attribution Error: Extending Allport's Cognitive Analysis of Prejudice, in: Personality and Social Psychology Bulletin 5: 4, 461-476.

Pouliot, Vincent 2008: The Logic of Practicality: A Theory of Practice of Security Communities, in: International Organization 62: 2, 257-288.

Preisendörfer, Peter 1995: Vertrauen als soziologische Kategorie, in: Zeitschrift für Soziologie 24: 4, 263-272.

Rathbun, Brian C. 2009: It Takes all Types: Social Psychology, Trust, and the International Relations Paradigm in our Minds, in: International Theory 1: 3, 345-380.

Rathbun, Brian C. 2011a: Before Hegemony: Generalized Trust and the Creation and Design of International Security Organizations, in: International Organization 65: 2, 243-273.

Rathbun, Brian C. 2011 b: From Vicious to Virtuous Circle: Moralistic Trust, Diffuse Reciprocity, and the American Security Commitment to Europe, in: European Journal of International Relations 18: 2, 323-344.

Rathbun, Brian C. 2011c: The >Magnificent Fraud «: Trust, International Cooperation, and the Hidden Domestic Politics of American Multilateralism after World War II, in: International Studies Quarterly 55: 1, 1-21.

Raudsepp, Maaris/Wagner, Wolfgang 2012: The Essentially Other: Representational Processes that Divide Groups, in: Marková, Ivana/Gillespie, Alex (Hrsg.): Trust and Conflict: Representation, Culture and Dialogue, New York, NY, 105-122.

Ripperger, Tanja 1998: Ökonomik des Vertrauens. Analyse eines Organisationsprinzips, Tübingen. 
Risse, Thomas 2007: Global Govenance und kommunikatives Handeln, in: Niesen, Peter/ Herborth, Benjamin (Hrsg.): Anarchie der kommunikativen Freiheit. Jürgen Habermas und die Theorie der internationalen Politik, Frankfurt a. M., 57-86.

Risse-Kappen, Thomas 1995: Democratic Peace - Warlike Democracies? A Social Constructivist Interpretation of the Liberal Argument, in: European Journal of International Relations 1: 4, 491-517.

Rotter, Julian B. 1967: A New Scale for the Measurement of Interpersonal Trust, in: Journal of Personality 35: 4, 651-665.

Rotter, Julian B. 1980: Interpersonal Trust, Trustworthiness, and Gullibility, in: American Psychologist 35: 1, 1-7.

Rousseau, Denise M./Sitkin, Sim B./Burt, Ronald S./Camerer, Colin 1998: Not so Different at all: A Cross-Discipline View of Trust, in: Academy of Management Review 23: 3, 393-404.

Rubini, Monica/Palmonari, Augusto 2012: Different and yet Human: Categorization and the Antecedents of Intergroup Trust, in: Marková, Ivana/Gillespie, Alex (Hrsg.): Trust and Conflict: Representation, Culture and Dialogue, New York, NY, 73-82.

Savage, Leonard J. 1954: The Foundations of Statistics, New York, NY.

Schweer, Martin K. 2012: Vertrauen als Organisationsprinzip in interorganisationalen Kooperationen, in: Schilcher, Christian/Will-Zoloch, Mascha/Ziegler, Marc (Hrsg.): Vertrauen und Kooperation in der Arbeitswelt, Wiesbaden, 103-121.

Siegenthaler, Hansjörg 1993: Regelvertrauen, Prosperität und Krisen, Tübingen.

Simmel, Georg 1983: Soziologie. Untersuchungen über die Formen der Vergesellschaftung, Berlin.

Stolle, Dietlind 2002: Trusting Strangers - The Concept of Generalized Trust in Perspective, in: Österreichische Zeitschrift für Politikwissenschaften 31: 4, 397-412.

Svendsen, Lars 2008: A Philosophy of Fear, London.

Sydow, Jörg 2006: How Can Systems Trust Systems? A Structuration Perspective on TrustBuilding in Inter-Organizational Relations, in: Bachmann, Reinhard/Zaheer, Akbar (Hrsg.): Handbook of Trust Research, Cheltenham, 377-392.

Taylor, Donald M./Jaggi, Vaishna 1974: Ethnocentrism and Causal Attribution in a South Indian Context, in: Journal of Cross-Cultural Psychology 5: 2, 162-171.

Uslaner, Eric M. 2002: The Moral Foundations of Trust, Cambridge.

Väyrynen, Raimo 2000: Stable Peace through Security Communities? Steps Towards TheoryBuilding, in: Kacowicz, Arie M./Bar-Siman-Tov, Yaacov/Jerneck, Magnus (Hrsg.): Stable Peace among Nations, Lanham, MD, 108-129.

Wendt, Alexander 1999: Social Theory of International Politics, Cambridge.

Wessells, Michael 2004: Diplomacy in the Era of Intrastate Conflict: Challenges of Transforming Cultures of Violence in to Cultures of Peace, in: Langholtz, Harvey J./Stout, Chris E. (Hrsg.): The Psychology of Diplomacy, Westport, 79-96.

Wheeler, Nicholas J. 2009: Beyond Waltz's Nuclear World: More Trust May be Better, in: International Relations 23: 3, 428-445.

Wheeler, Nicholas J. 2010: »I Had Gone to Lahore With a Message of Goodwill But in Return We Got Kargil«: The Promise and Perils of »Leaps of Trust« in India-Pakistan Relations, in: India Review 9: 3, 319-344.

Williams, Michele 2001: In Whom We Trust: Group Membership as an Affective Context for Trust Development, in: The Academy of Management Review 26: 3, 377-396.

Williamson, Oliver E. 1993: Calculativeness, Trust, and Economic Organization, in: The Journal of Law \& Economics 36: 1, 453-486.

Wolf, Reinhard 2008: Respekt: Ein unterschätzter Faktor in den Internationalen Beziehungen, in: Zeitschrift für Internationale Beziehungen 15: 1, 5-42.

Wolf, Reinhard 2011: Respect and Disrespect in International Politics: The Significance of Status Recognition, in: International Theory 3: 1, 105-142. 
Yamagishi, Toshio/Kanazawa, Satoshi/Mashima, Rie/Terai, Shigeru 2005: Separating Trust from Cooperation in a Dynamic Relationship: Prisoner's Dilemma with Variable Dependence, in: Rationality and Society 17: 3, 275-308.

Yamagishi, Toshio/Kikuchi, Masako/Kosugi, Motoko 1999: Trust, Gullibility, and Social Intelligence, in: Asian Journal of Social Psychology 2: 1, 145-161.

Yamagishi, Toshio/Kiyonari, Toko 2000: The Group as the Container of Generalized Reciprocity, in: Social Psychology Quarterly 63: 2, 116-132.

Yamagishi, Toshio/Yamagishi, Midori 1994: Trust and Commitment in the United States and Japan, in: Motivation and Emotion 18: 2, 129-166.

Zaheer, Akbar/Harris, Jared 2006: Interorganizational Trust, in: Shenkar, Oded/Reuer, Jeffrey J. (Hrsg.): Handbook of Strategic Alliances, Thousand Oaks, CA, 169-197.

Zaheer, Akbar/McEvily, Bill/Perrone, Vincenzo 1998: Does Trust Matter? Exploring the Effects of Interorganizational and Interpersonal Trust on Performance, in: Organization Science 9: 2, 141-159.

Zak, Paul J./Kugler, Jacek 2011: Neuroeconomics and International Studies: A New Understanding of Trust, in: International Studies Perspectives 12: 2, 136-152. 